\title{
Selective Preparation and High Dynamic-Range Analysis of Cannabinoids in "CBD Oil" and Other Cannabis sativa Preparations
}

Takashi Ohtsuki, J. Brent Friesen, Shao-Nong Chen, James B. McAlpine, and Guido F. Pauli*

\section{- SUPPORTING INFORMATION}




\section{- TABLE OF CONTENTS}

No. Content Page

S1 1H NMR of CBD and related cannabinoids in CBD oil $\quad$ S-4

S2 $\quad{ }^{1}$ H NMR of Upper Phase (HAcM extractions) S-5

S3 $\quad{ }^{1} \mathrm{H}$ NMR of Lower Phase (HAcM extractions) $\quad$ S-6

$\begin{array}{lll} & { }^{1} \mathrm{H} \text { NMR spectrum of CBD (1) } & \text { S-7 }\end{array}$

S5 $\quad{ }^{1} \mathrm{H}$ NMR spectrum of trans- $\Delta^{9}$-THC (2b) S-8

$\begin{array}{lll} & { }^{1} \mathrm{H} \text { NMR spectrum of } c i s-\Delta^{9}-\mathrm{THC}(\mathbf{2 a}) & \text { S-9 }\end{array}$

$\begin{array}{lll}\text { S7 } & { }^{1} \mathrm{H} \text { NMR spectrum of CBC (3) } & \text { S-10 }\end{array}$

$\begin{array}{lll}{ }^{1} \mathrm{H} & \mathrm{NMR} \text { spectrum of } \mathrm{CBG}(4) & \mathrm{S}-11\end{array}$

$\begin{array}{lll}{ }^{1} \mathrm{H} & \mathrm{NMR} \text { spectrum of } \mathrm{CBN}(\mathbf{5}) & \mathrm{S}-12\end{array}$

S10 $\quad{ }^{1} \mathrm{H}$ NMR spectrum of CBDV (6) S-13

$\begin{array}{lll}{ }^{1} \mathrm{H} & \mathrm{NMR} \text { COSY spectrum of CBC (3) } & \text { S-14 }\end{array}$

S12 ${ }^{1} \mathrm{H} /{ }^{13} \mathrm{C}$ NMR HSQC spectrum of CBC (3) S-15

S13 $\quad{ }^{1} \mathrm{H} /{ }^{13} \mathrm{C}$ NMR HMBC spectrum of CBC (3) S-16

$\begin{array}{lll}{ }^{1} \mathrm{H} & \mathrm{NMR} \text { NOESY spectrum of CBC (3) } & \text { S-17 }\end{array}$

$\begin{array}{lll}{ }^{1} \mathrm{H} & \mathrm{NMR} \text { NOESY spectrum of CBC (3) } & \text { S-18 }\end{array}$

$\begin{array}{ll}\text { S16 High-frequency region stacked plot of }{ }^{1} \mathrm{H} \text { NMR spectra } & \text { S-19 }\end{array}$

S17 Low-frequency region stacked plot of ${ }^{1} \mathrm{H}$ NMR spectra $\quad$ S-20

S18 High-frequency region stacked plot of ${ }^{1} \mathrm{H}$ NMR spectra $\quad$ S-21 
S19 Low-frequency region stacked plot of ${ }^{1} \mathrm{H}$ NMR spectra

S20 High-frequency region stacked plot of ${ }^{1} \mathrm{H}$ NMR spectra $\quad$ S-23

$\begin{array}{lll}\text { S21 Low-frequency region stacked plot of }{ }^{1} \mathrm{H} \text { NMR spectra } & \text { S-24 }\end{array}$

S22 High-frequency region stacked plot of ${ }^{1} \mathrm{H}$ NMR spectra $\quad$ S-25

$\begin{array}{lll}\text { S23 Low-frequency region stacked plot of }{ }^{1} \mathrm{H} \text { NMR spectra } & \text { S-26 }\end{array}$

$\begin{array}{lll}{ }^{1} \mathrm{H} & \text { NMR spectrum of fraction JK-H } & \text { S-27 }\end{array}$

S25 ${ }^{13}$ C NMR Spectrum of CBD (1) S-28

$\begin{array}{lll}\text { S26 } & { }^{13} \text { C NMR Spectrum of trans- } \Delta^{9} \text {-THC (2a) } & \text { S-29 }\end{array}$

S27 ${ }^{13}$ C NMR Spectrum of $c$ is $-\Delta^{9}$-THC (2b) S-30

S28 $\quad{ }^{13}$ C NMR Spectrum of CBC (3) S-31

$\begin{array}{lll}\text { S29 } & { }^{13} \text { C NMR Spectrum of CBG (4) S-32 }\end{array}$

S30 $\quad{ }^{13}$ C NMR Spectrum of CBN (5) S-33

S31 ${ }^{13}$ C NMR Spectrum of CBDV (6) S-34

S32 EC-qHNMR calibration curve for $\mathrm{DMSO}_{2} \quad \mathrm{~S}-35$

S33 The ${ }^{1} \mathrm{H}$ iterative full spin analysis (HiFSA) profiles of CBD (1) S-36

S34 The ${ }^{1} \mathrm{H}$ iterative full spin analysis (HiFSA) profiles of trans $-\Delta^{9}-\mathrm{THC}(\mathbf{2 a}) \quad$ S-37

S35 The ${ }^{1} \mathrm{H}$ iterative full spin analysis (HiFSA) profiles of $c i s-\Delta^{9}-\mathrm{THC}(\mathbf{2 b}) \quad$ S-38

S36 The ${ }^{1} \mathrm{H}$ iterative full spin analysis (HiFSA) profiles of CBC (3) $\quad$ S-39

S37 The ${ }^{1} \mathrm{H}$ iterative full spin analysis (HiFSA) profiles of CBG (4) S-40

S38 The ${ }^{1} \mathrm{H}$ iterative full spin analysis (HiFSA) profiles of CBDV (6) S-41

S39 The ${ }^{1} \mathrm{H}$ iterative full spin analysis (HiFSA) profiles of CBN (5) S-42 
Figure S1. ${ }^{1} \mathrm{H}$ NMR of CBD (1) and related cannabinoids in "CBD Oil" $\left(400 \mathrm{MHz}, \mathrm{CDCl}_{3}\right)$.

\section{${ }^{1} \mathrm{H}$ NMR of CBD oil}
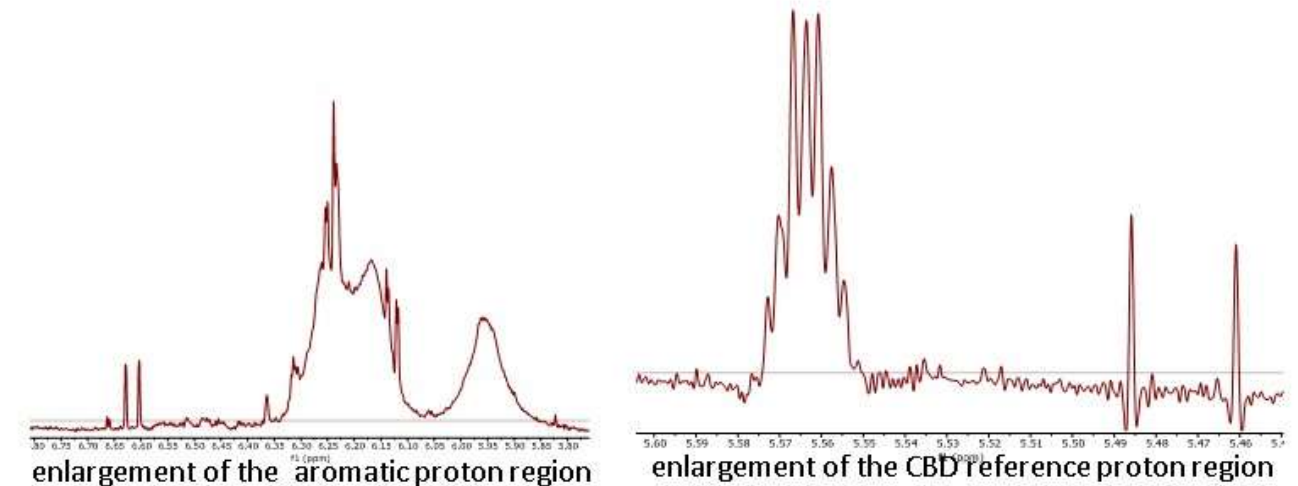

enlargement of the aromatic proton region

enlargement of the CBDTreference proton region

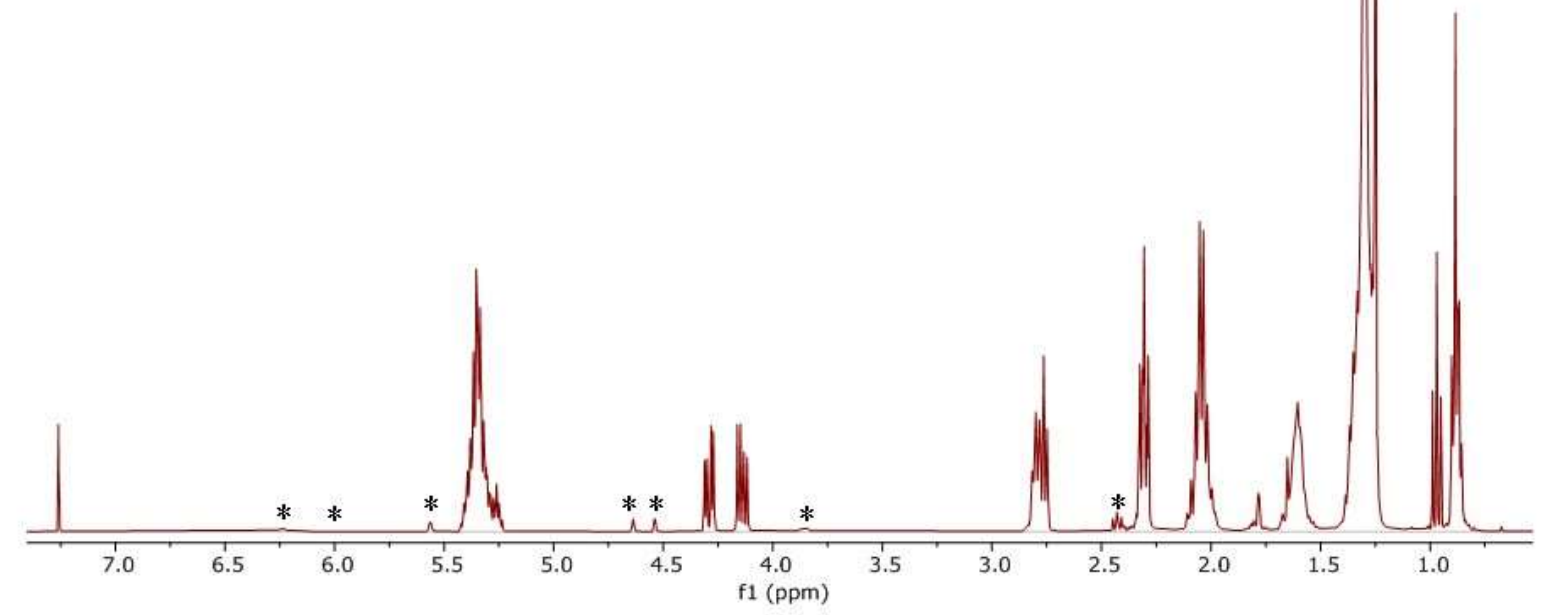


Figure S2. ${ }^{1} \mathrm{H}$ NMR of the Lower Phase (HAcM extractions) (400 MHz, $\left.\mathrm{CDCl}_{3}\right)$.

\section{${ }^{1} \mathrm{H}$ NMR of Lower Phase (HAcM extraction)}

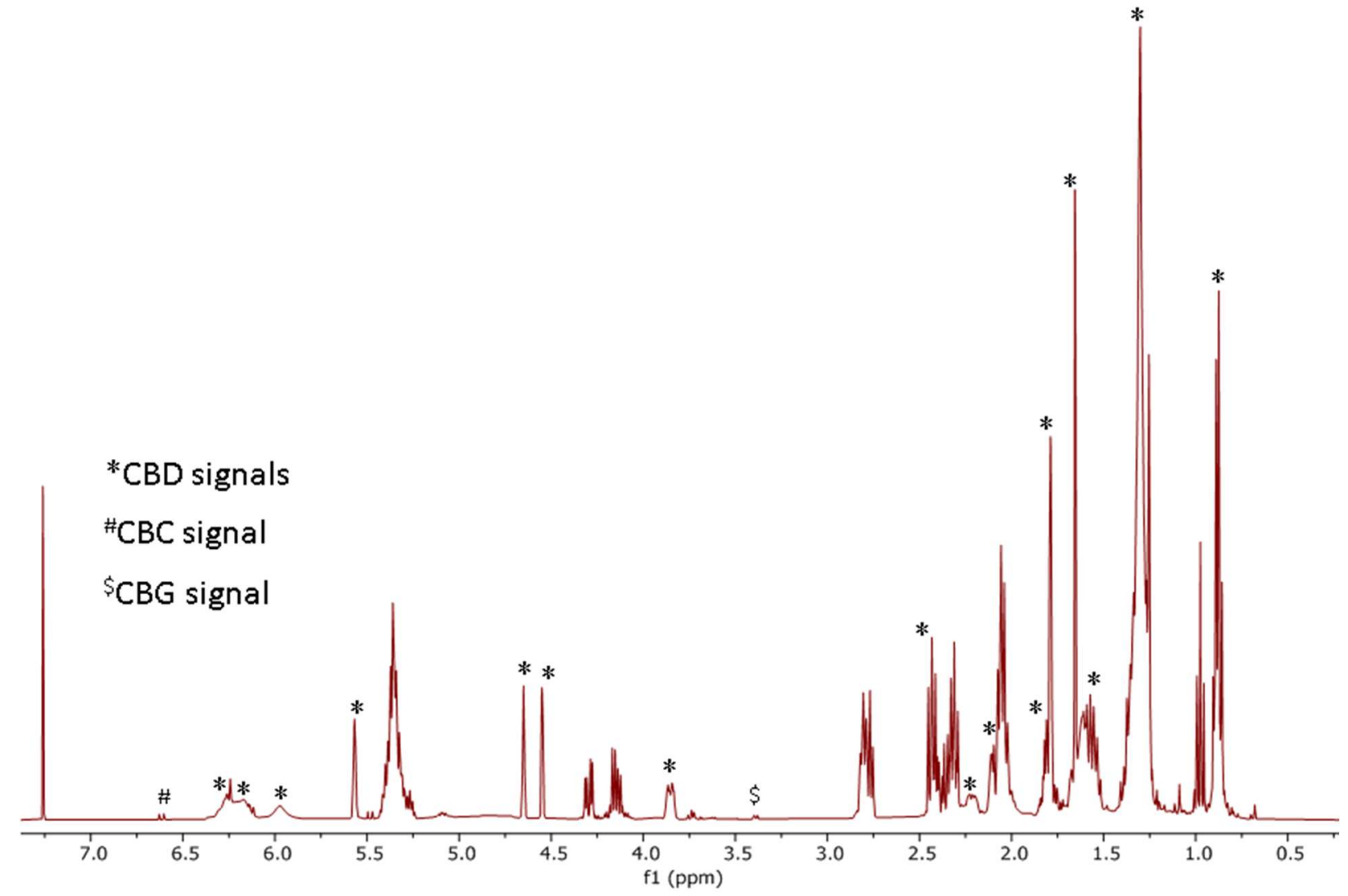


Figure S3. ${ }^{1} \mathrm{H}$ NMR of the Upper Phase (HAcM extractions) $\left(400 \mathrm{MHz}, \mathrm{CDCl}_{3}\right)$.

\section{${ }^{1} \mathrm{H}$ NMR of Upper Phase (HAcM extraction)}

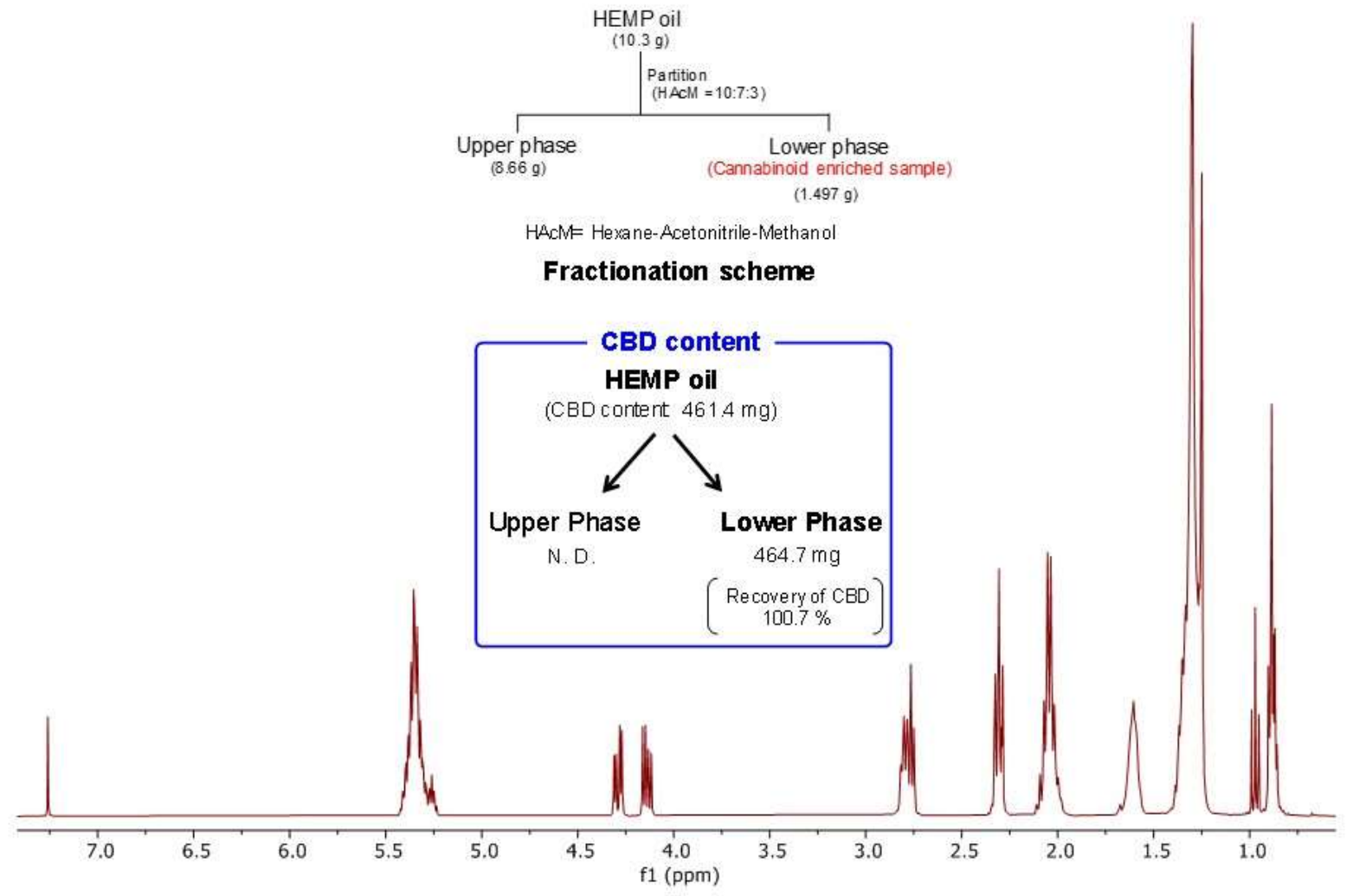


Figure S4. ${ }^{1} \mathrm{H}$ NMR Spectrum of CBD (1) (400 MHz, $\left.\mathrm{CDCl}_{3}\right)$.

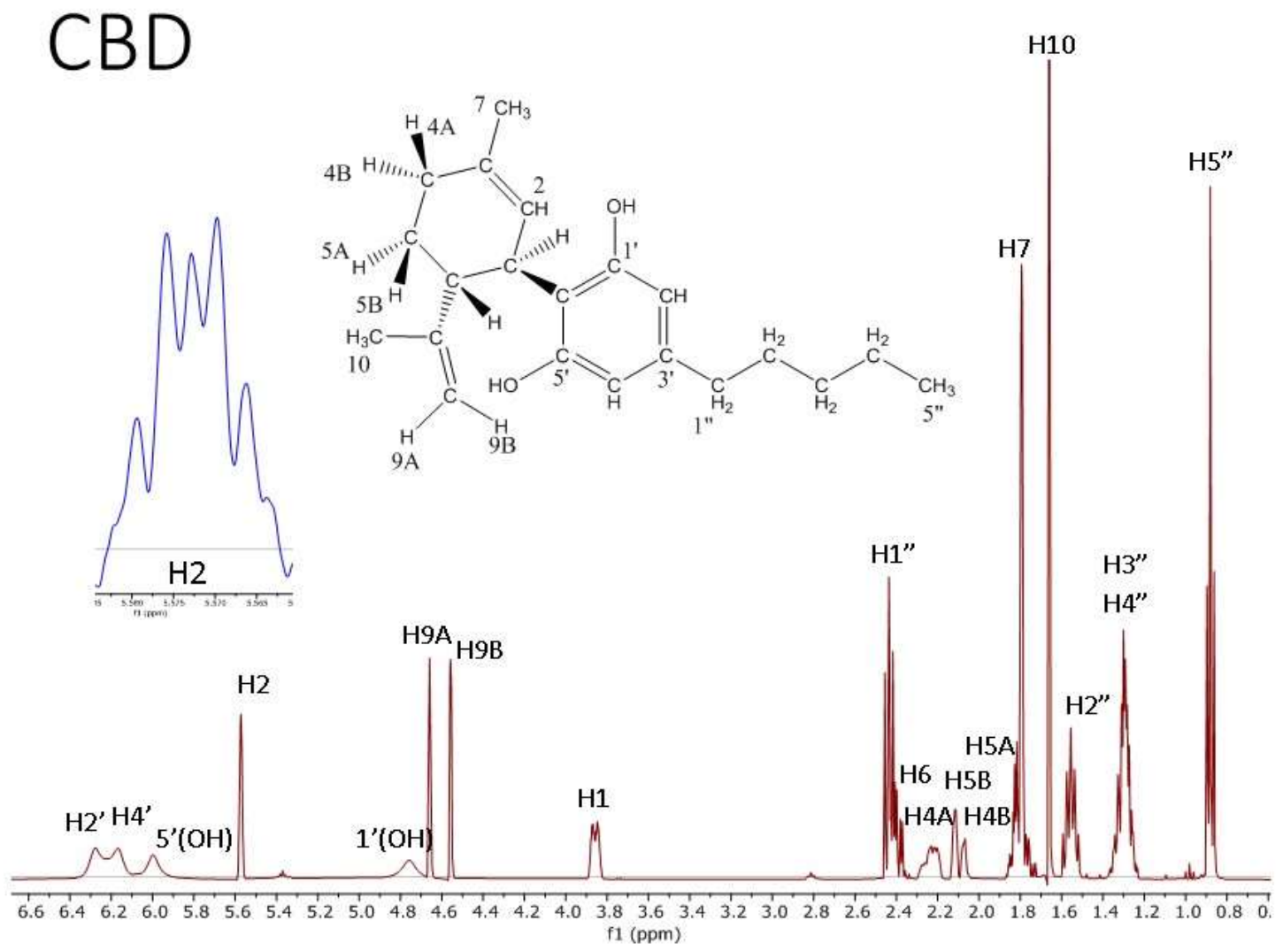


Figure S5. ${ }^{1} \mathrm{H}$ NMR Spectrum of trans- $\Delta^{9}-\mathrm{THC}(\mathbf{2 b})\left(400 \mathrm{MHz}, \mathrm{CDCl}_{3}\right)$.

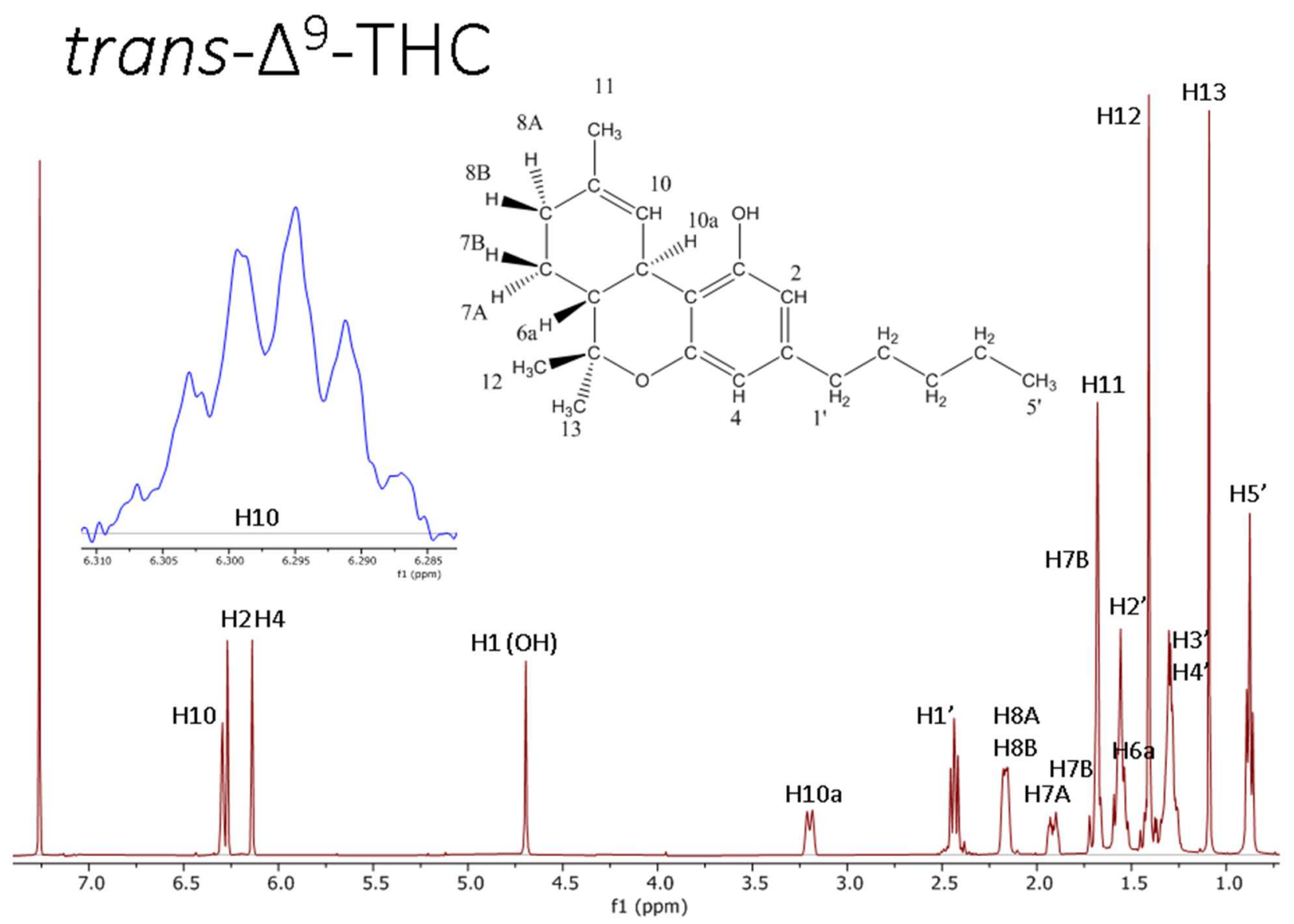


Figure S6. ${ }^{1} \mathrm{H}$ NMR Spectrum of $c i s-\Delta^{9}-\mathrm{THC}(\mathbf{2 a})\left(400 \mathrm{MHz}, \mathrm{CDCl}_{3}\right)$.

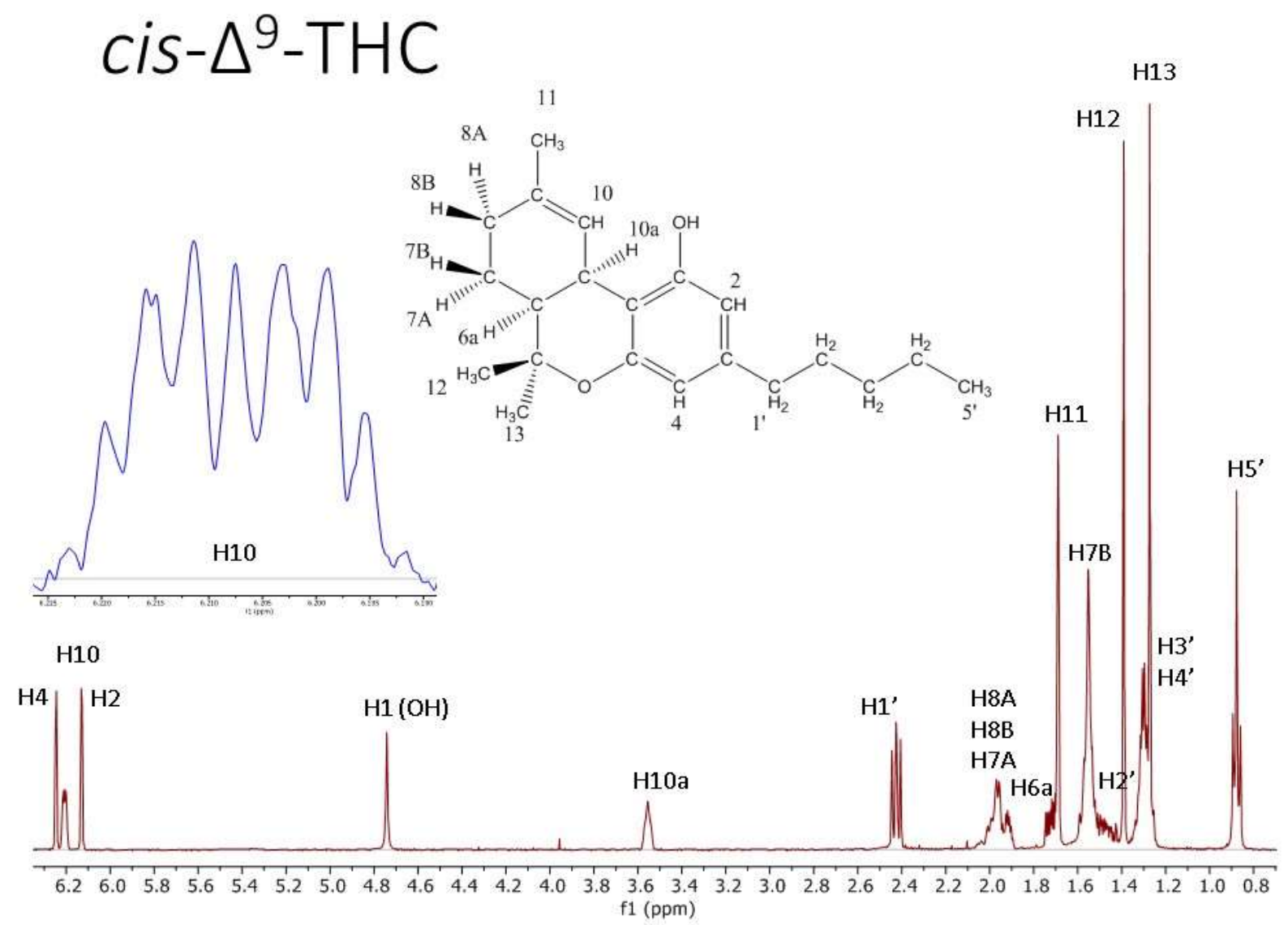


Figure S7. ${ }^{1} \mathrm{H}$ NMR Spectrum of $\mathrm{CBC}(3)\left(400 \mathrm{MHz}, \mathrm{CDCl}_{3}\right)$.

191111-Fr14-18-5_4600ug600uL_CDC13_Takashi_191120

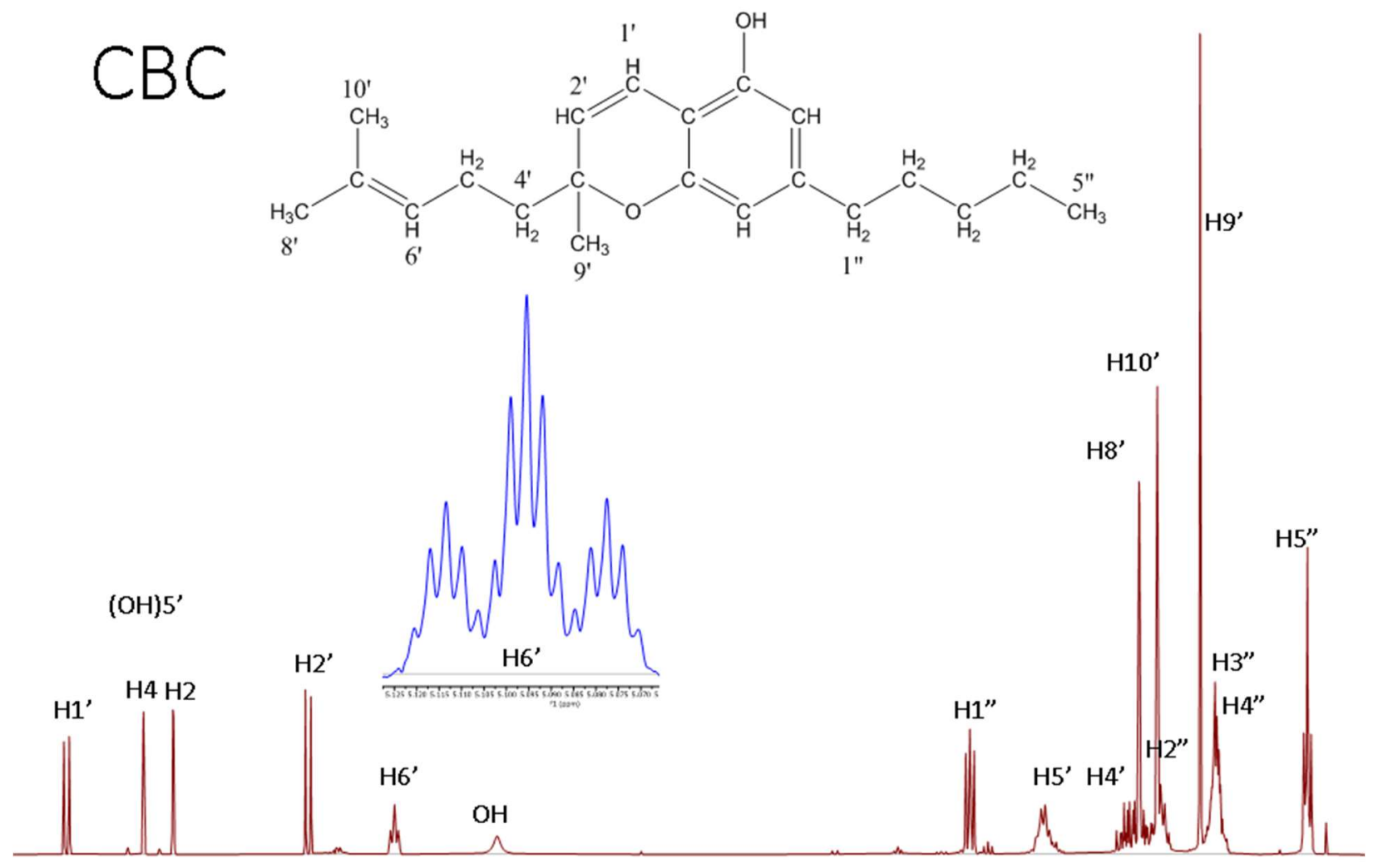

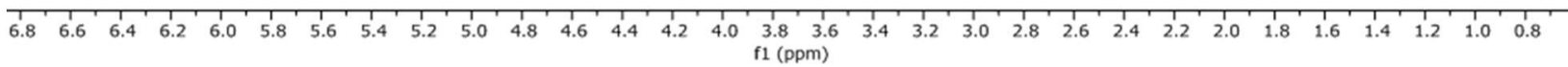


Figure S8. ${ }^{1} \mathrm{H}$ NMR Spectrum of CBG (4) (400 MHz, $\left.\mathrm{CDCl}_{3}\right)$.

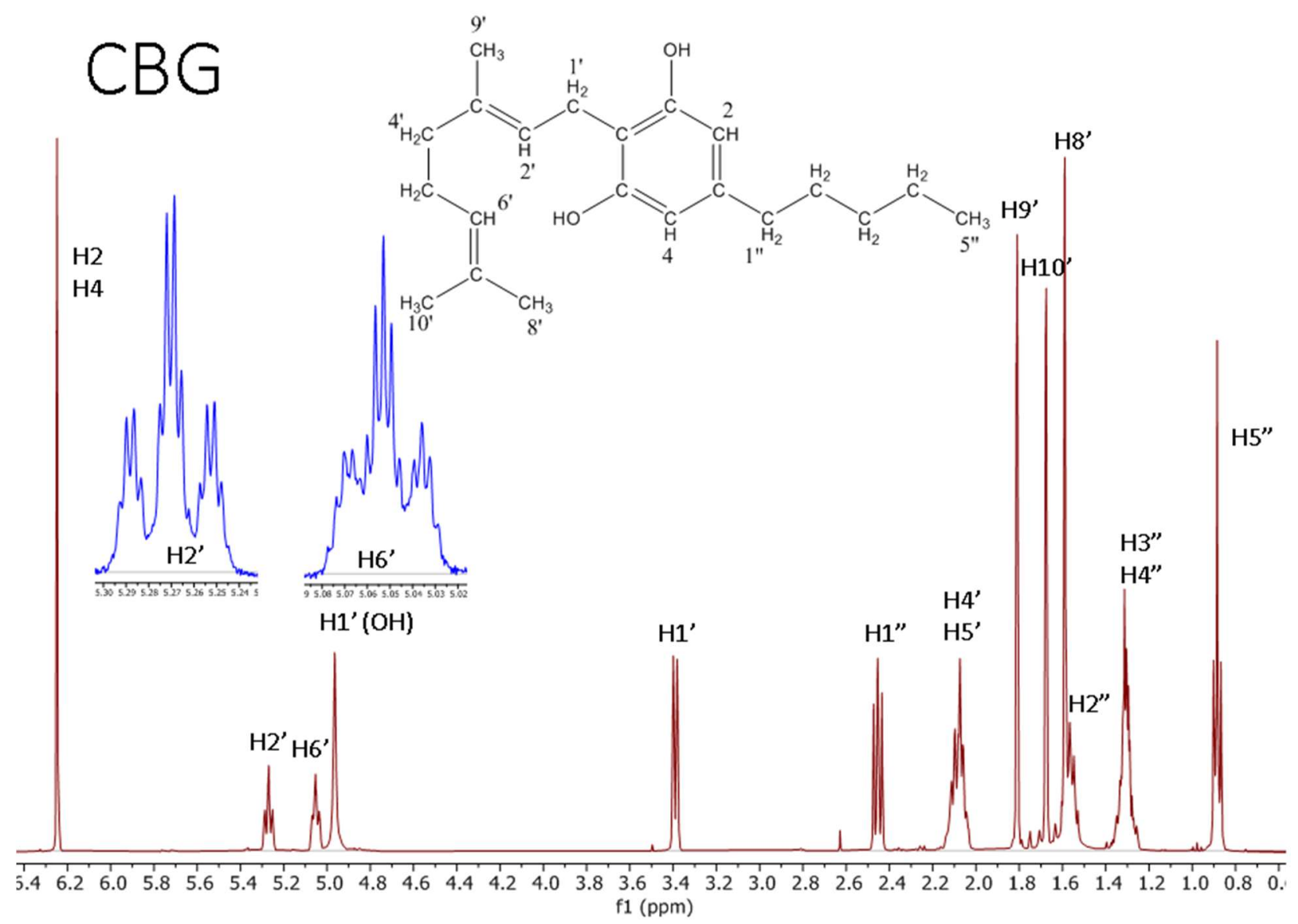


Figure S9. ${ }^{1} \mathrm{H}$ NMR Spectrum of CBN (5) $\left(400 \mathrm{MHz}, \mathrm{CDCl}_{3}\right)$.

cannabinoids_CBN_I50_600uL_CDC13_JBF_20210726 4

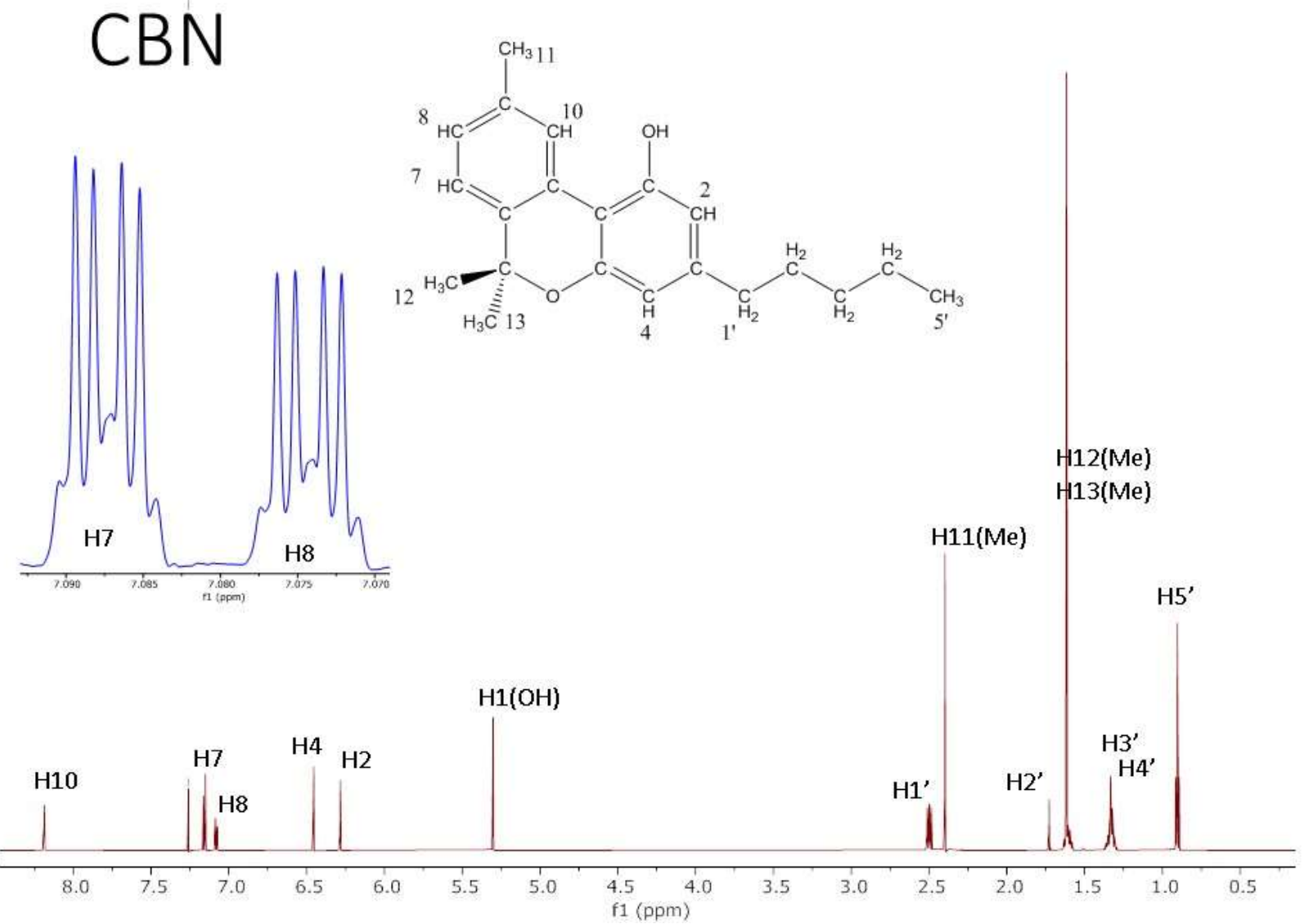


Figure S10. ${ }^{1} \mathrm{H}$ NMR Spectrum of CBDV (6) $\left(400 \mathrm{MHz}, \mathrm{CDCl}_{3}\right)$.

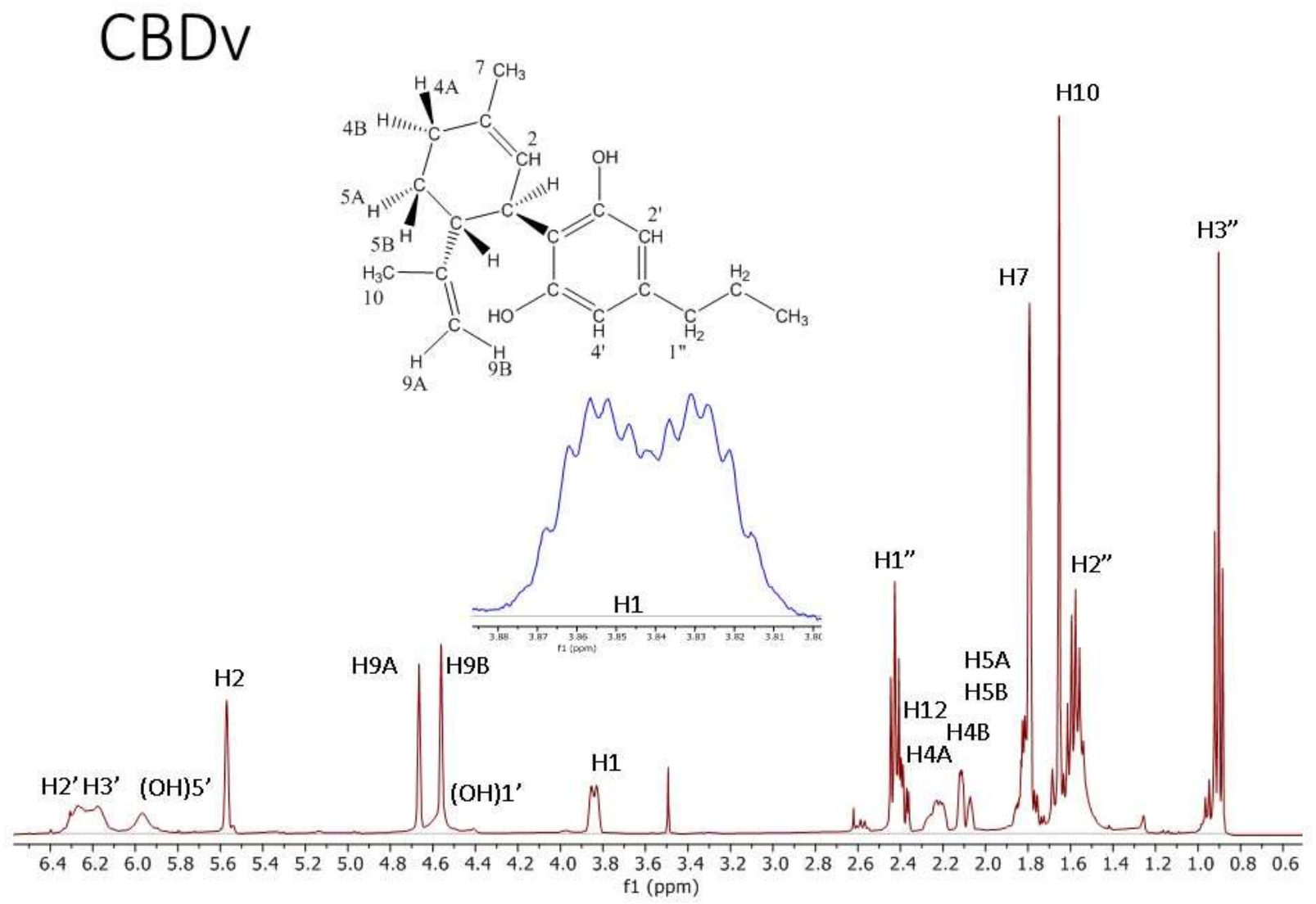


Figure S11. ${ }^{1} \mathrm{H}$ NMR COSY Spectrum of CBC (3) (400 MHz, $\left.\mathrm{CDCl}_{3}\right)$.

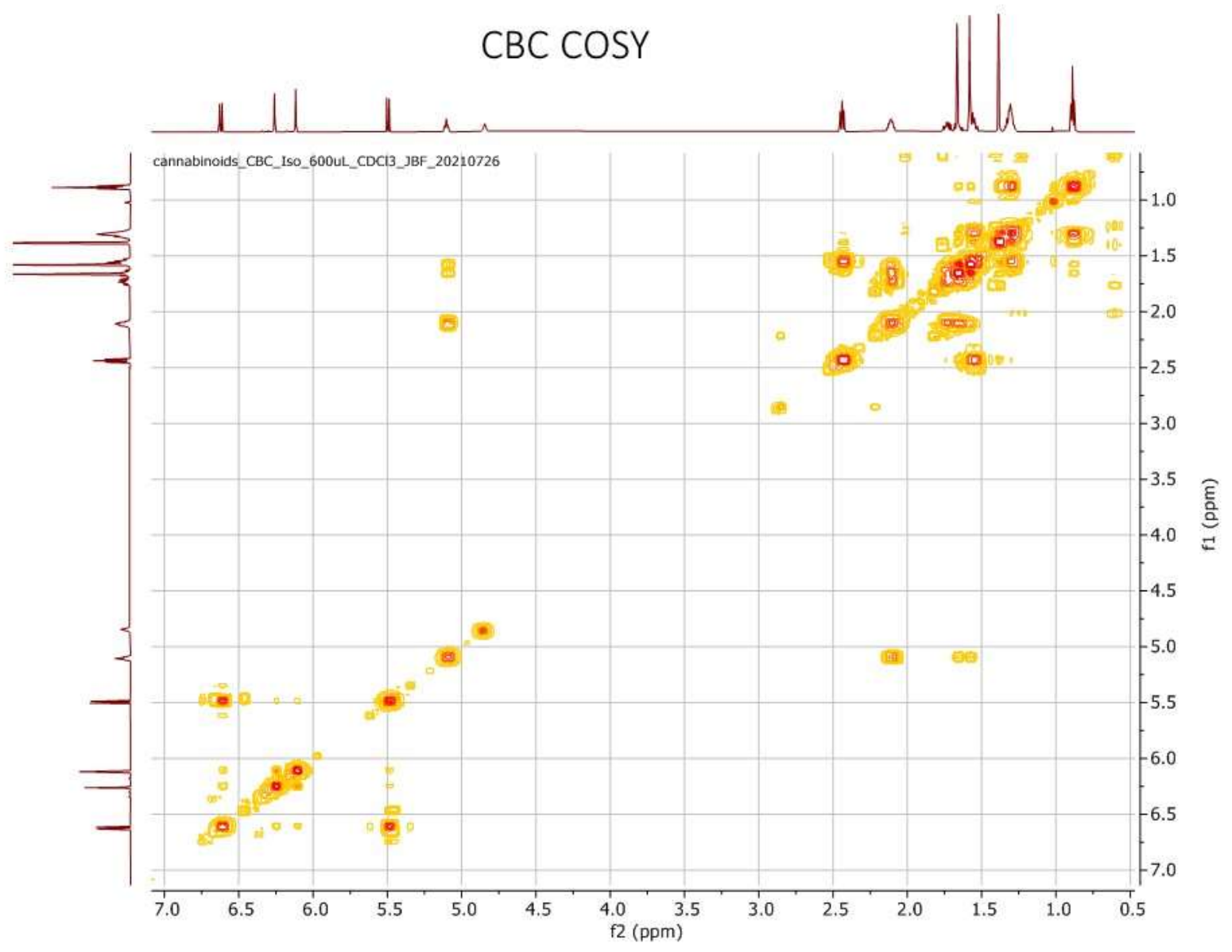


Figure S12. ${ }^{1} \mathrm{H} /{ }^{13} \mathrm{C}$ NMR HSQC Spectrum of CBC (3) $\left(400 / 100 \mathrm{MHz}, \mathrm{CDCl}_{3}\right)$.

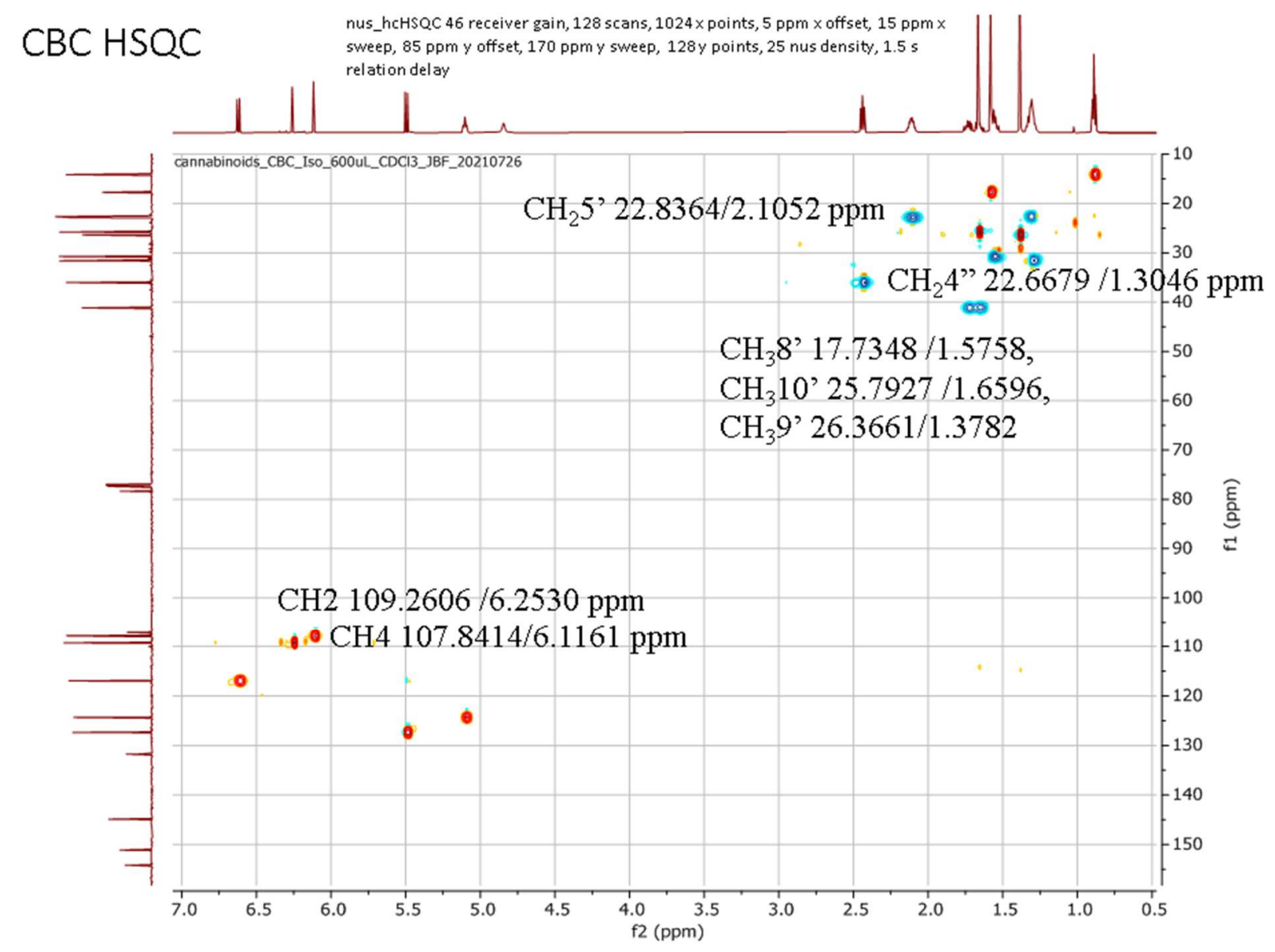


Figure S13. ${ }^{1} \mathrm{H} /{ }^{13} \mathrm{C}$ NMR HMBC Spectrum of CBC (3)(400/100 MHz, $\left.\mathrm{CDCl}_{3}\right)$.

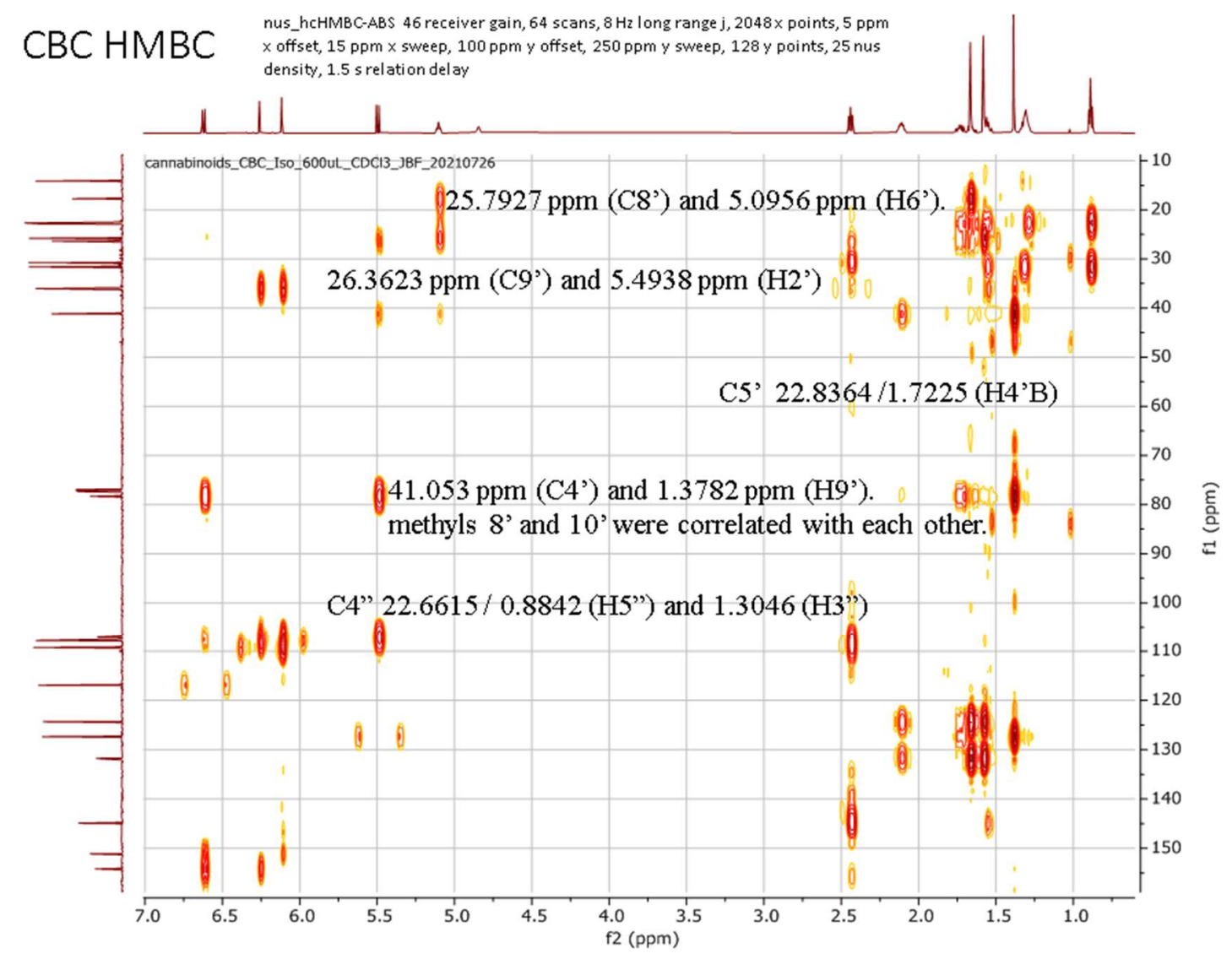


Figure S14. ${ }^{1} \mathrm{H}$ NMR NOESY Spectrum of CBC (3) $\left(400 \mathrm{MHz}, \mathrm{CDCl}_{3}\right)$.

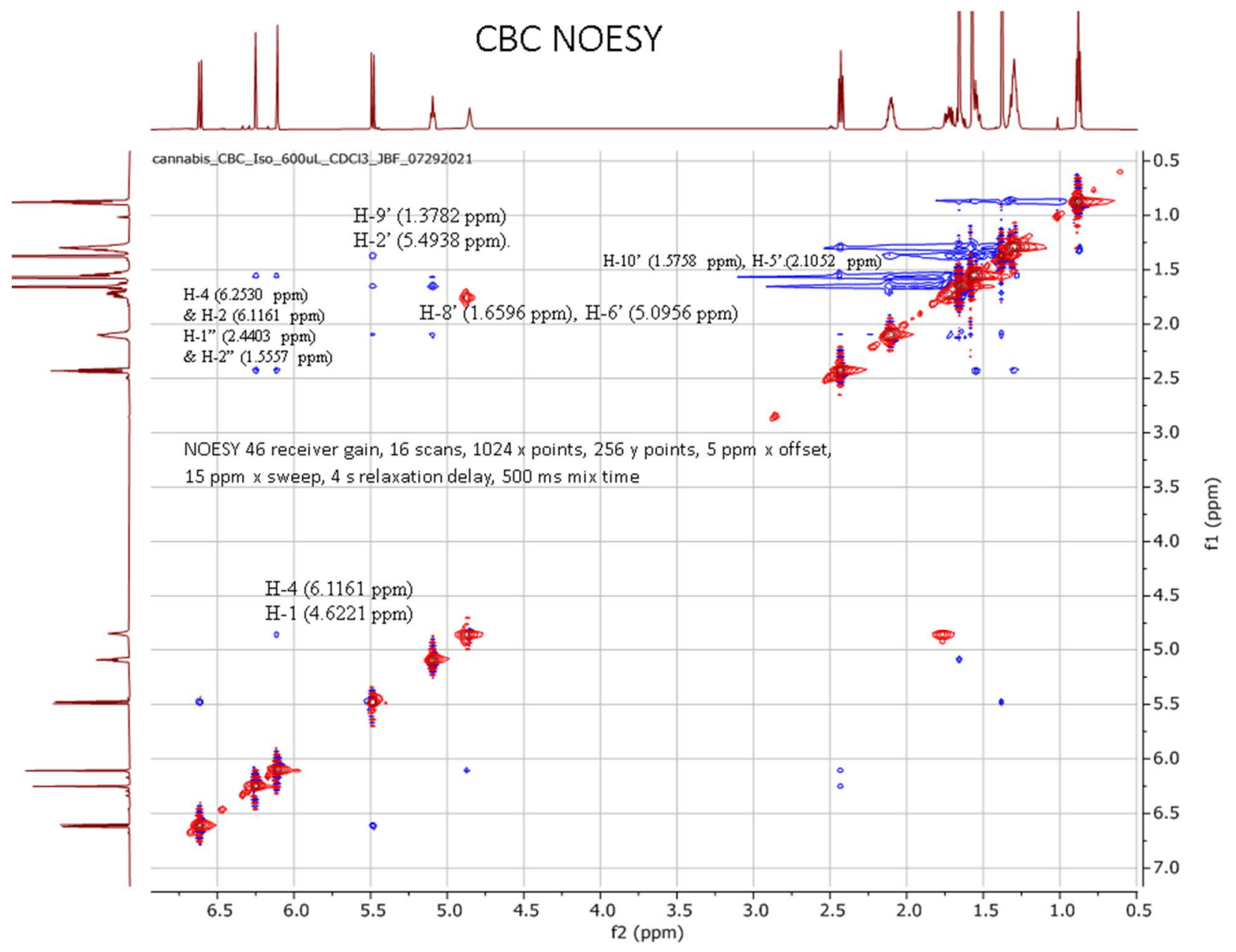


Figure S15. ${ }^{1} \mathrm{H}$ NMR NOESY Spectrum of CBC (3) $\left(400 \mathrm{MHz}, \mathrm{CDCl}_{3}\right)$.

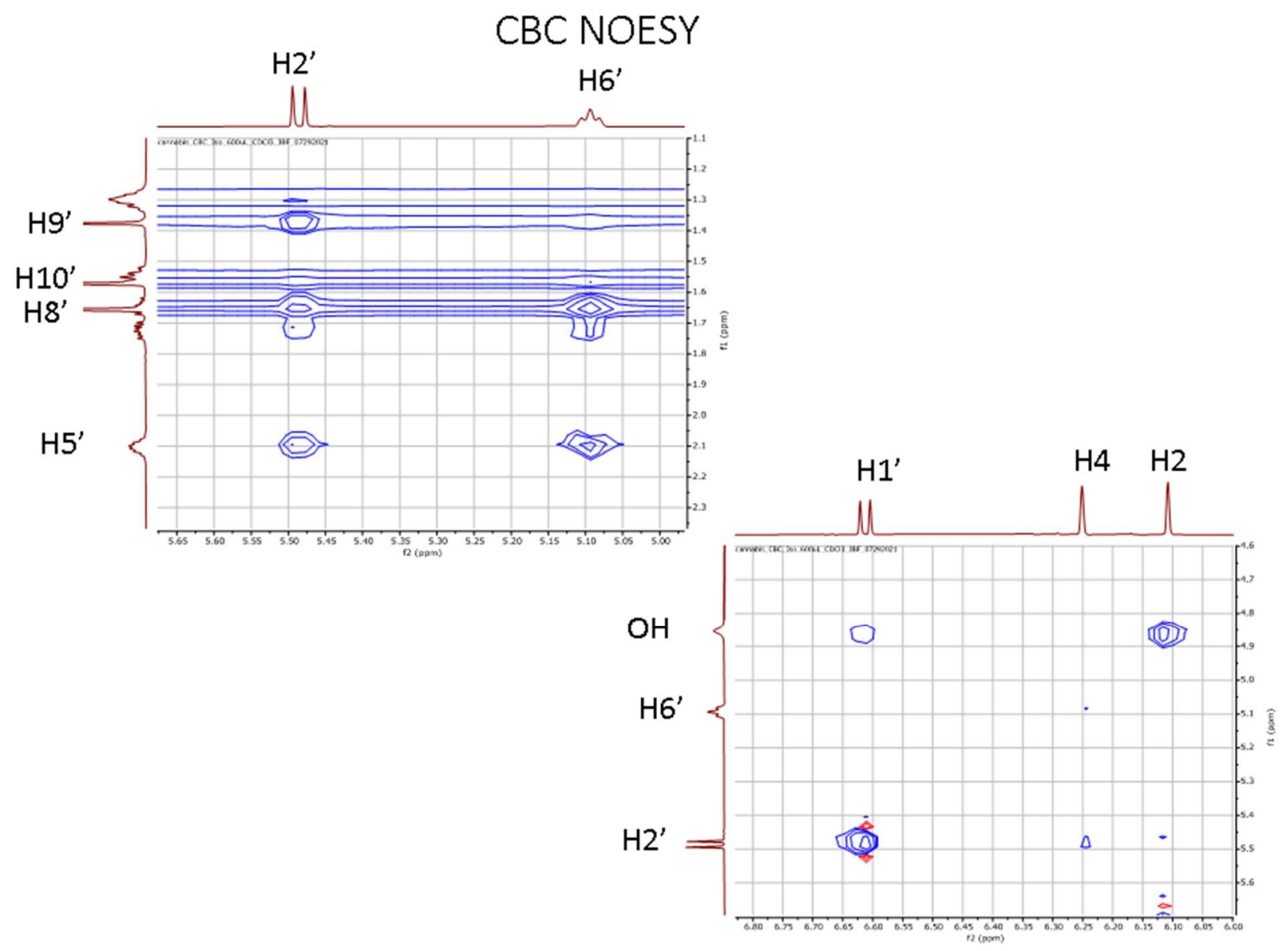


Figure S16. High-frequency region stacked plot of ${ }^{1} \mathrm{H}$ NMR spectra (400 $\mathrm{MHz}, \mathrm{CDCl}_{3}$ ).

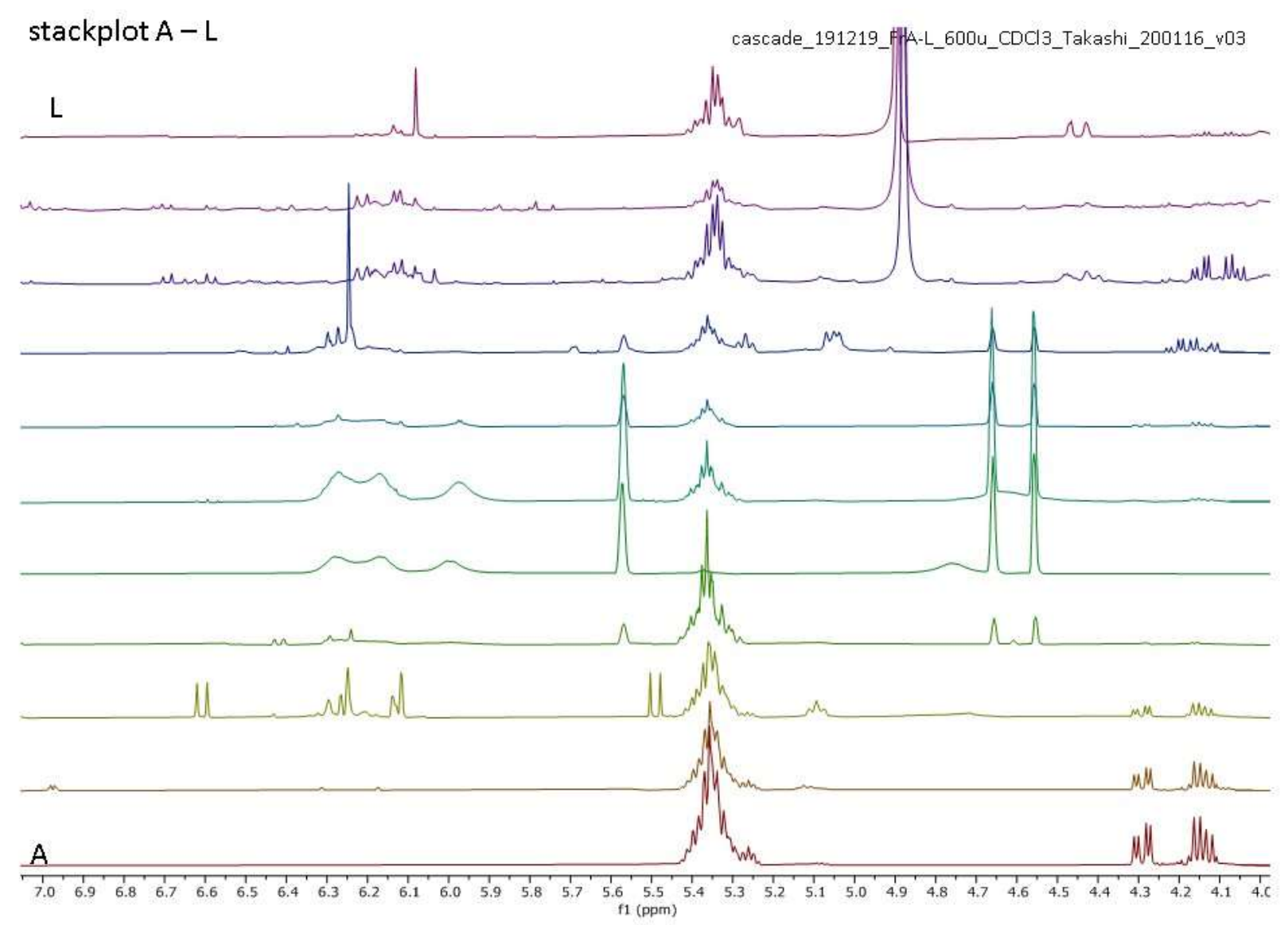


Figure S17. Low-frequency region stacked plot of ${ }^{1} \mathrm{H}$ NMR spectra (400 $\left.\mathrm{MHz}, \mathrm{CDCl}_{3}\right)$.

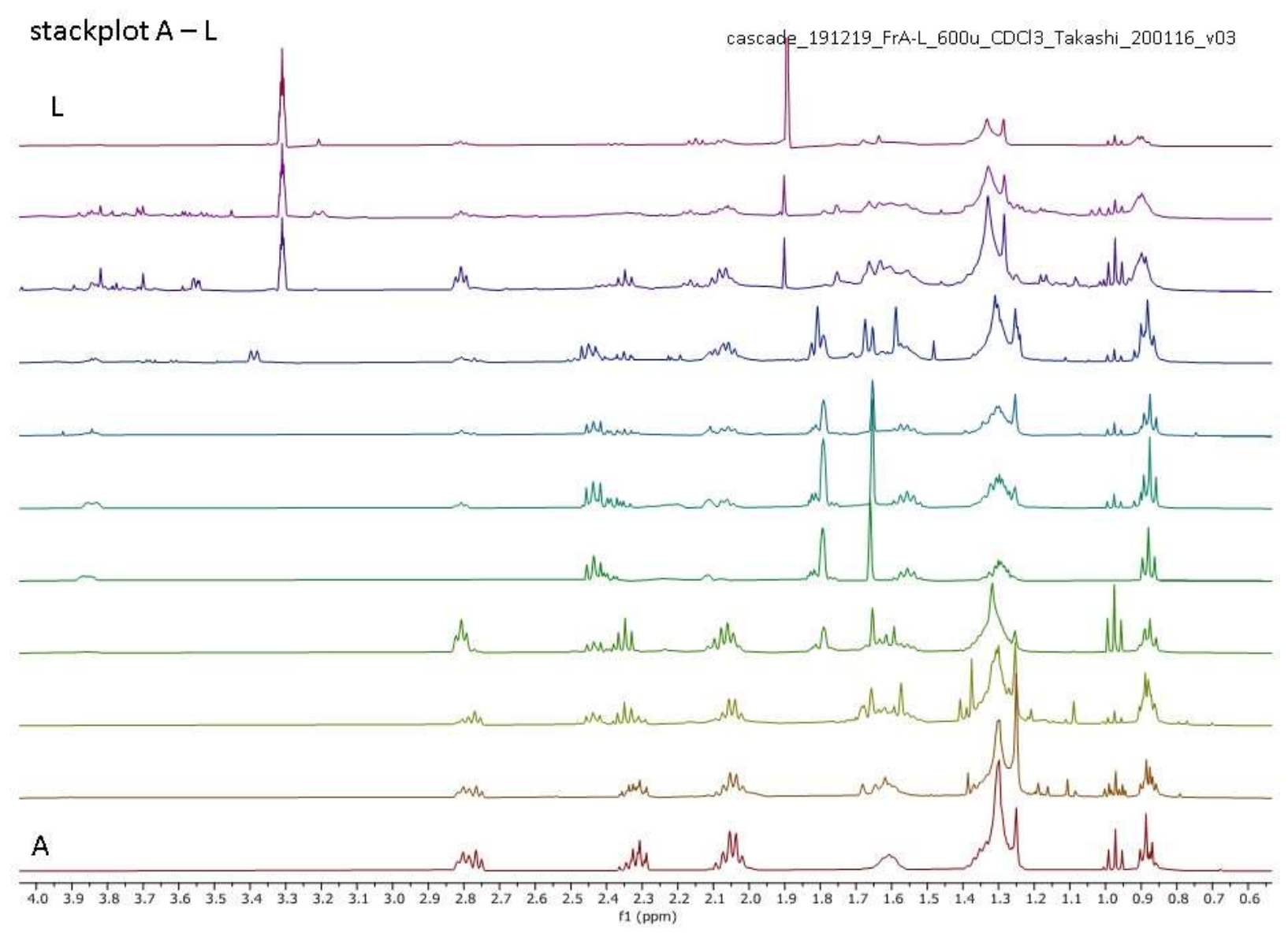


Figure S18. High-frequency region stacked plot of ${ }^{1} \mathrm{H}$ NMR spectra (400 $\mathrm{MHz}, \mathrm{CDCl}_{3}$ ).

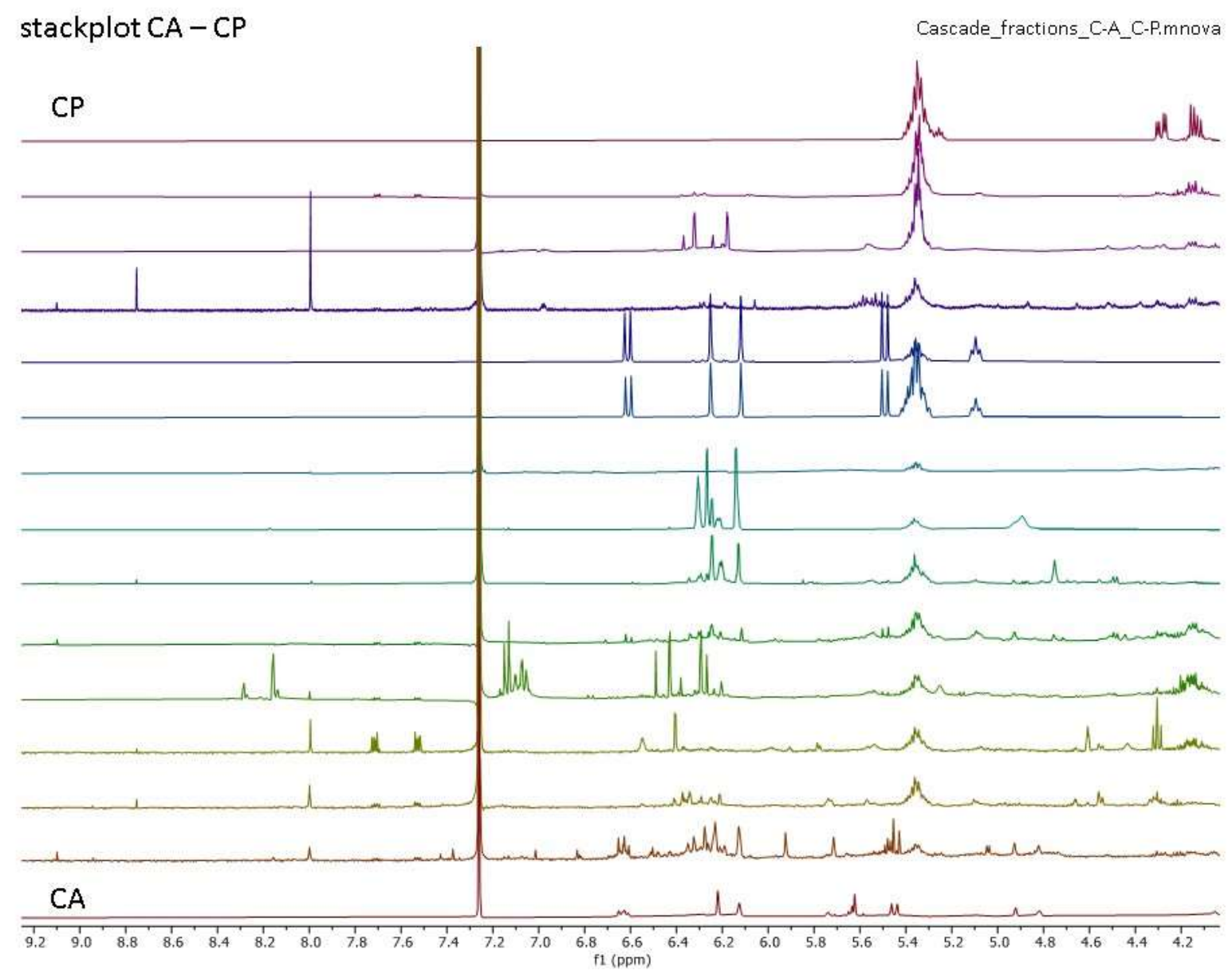


Figure S19. Low-frequency region stacked plot of ${ }^{1} \mathrm{H}$ NMR spectra (400 $\left.\mathrm{MHz}, \mathrm{CDCl}_{3}\right)$.

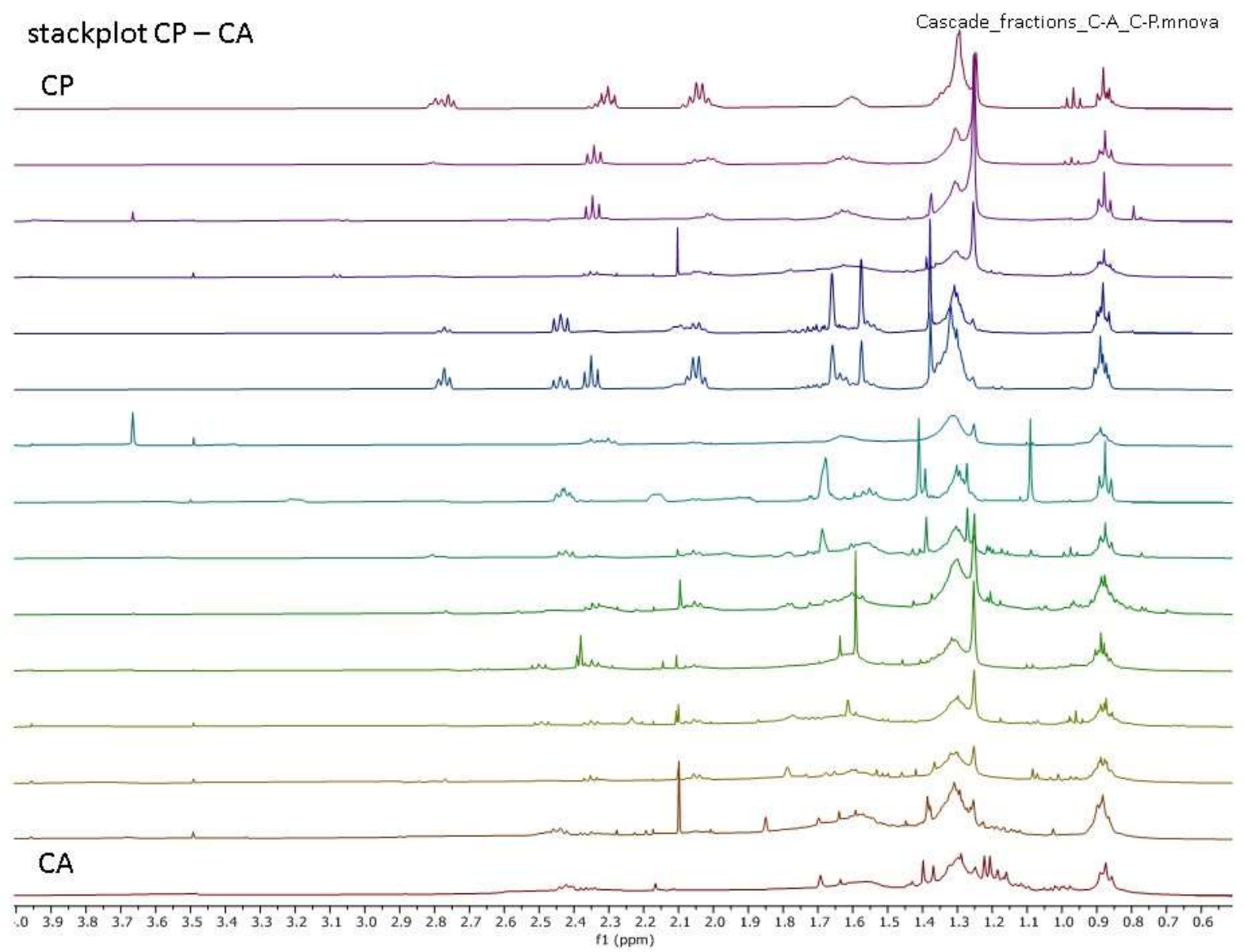


Figure S20. High-frequency region stacked plot of ${ }^{1} \mathrm{H}$ NMR spectra (400 $\mathrm{MHz}, \mathrm{CDCl}_{3}$ ).

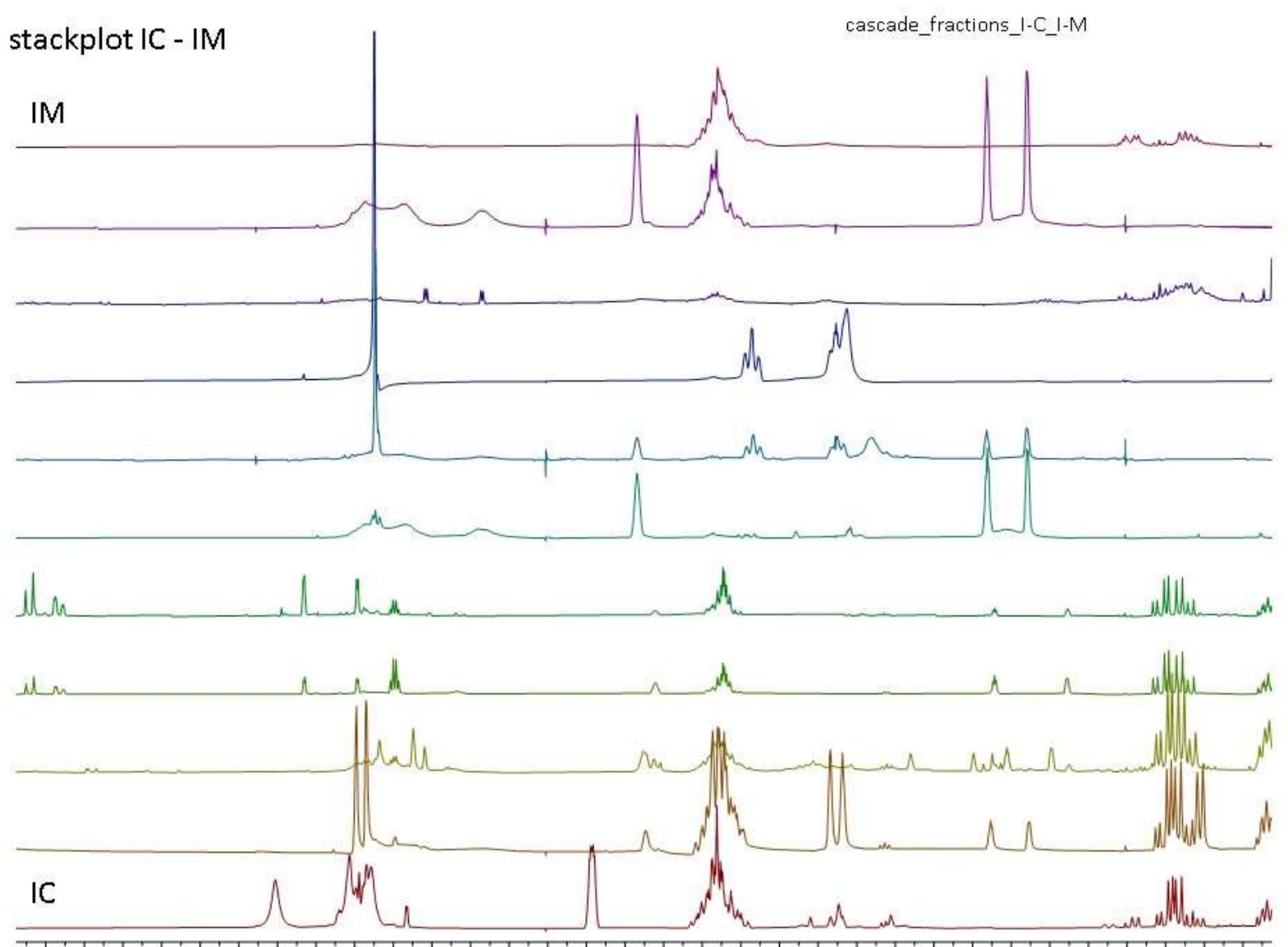

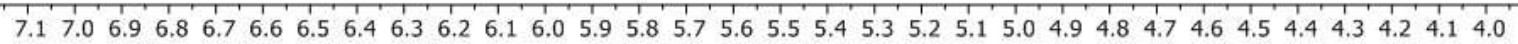
f1 (ppm) 
Figure S21. Low-frequency region stacked plot of ${ }^{1} \mathrm{H}$ NMR spectra (400 $\left.\mathrm{MHz}, \mathrm{CDCl}_{3}\right)$.

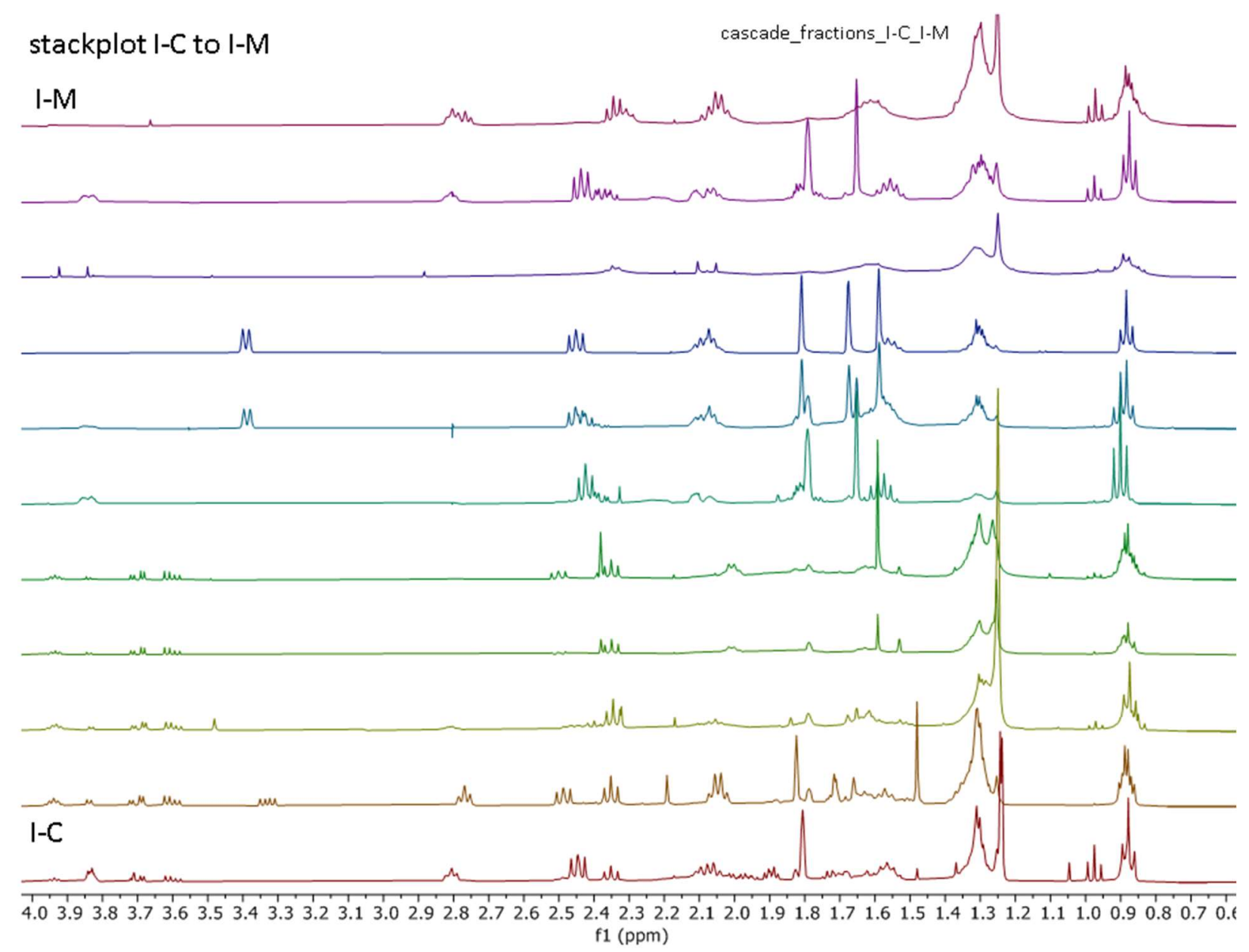


Figure S22. High-frequency region stacked plot of ${ }^{1} \mathrm{H}$ NMR spectra (400 $\mathrm{MHz}, \mathrm{CDCl}_{3}$ ).

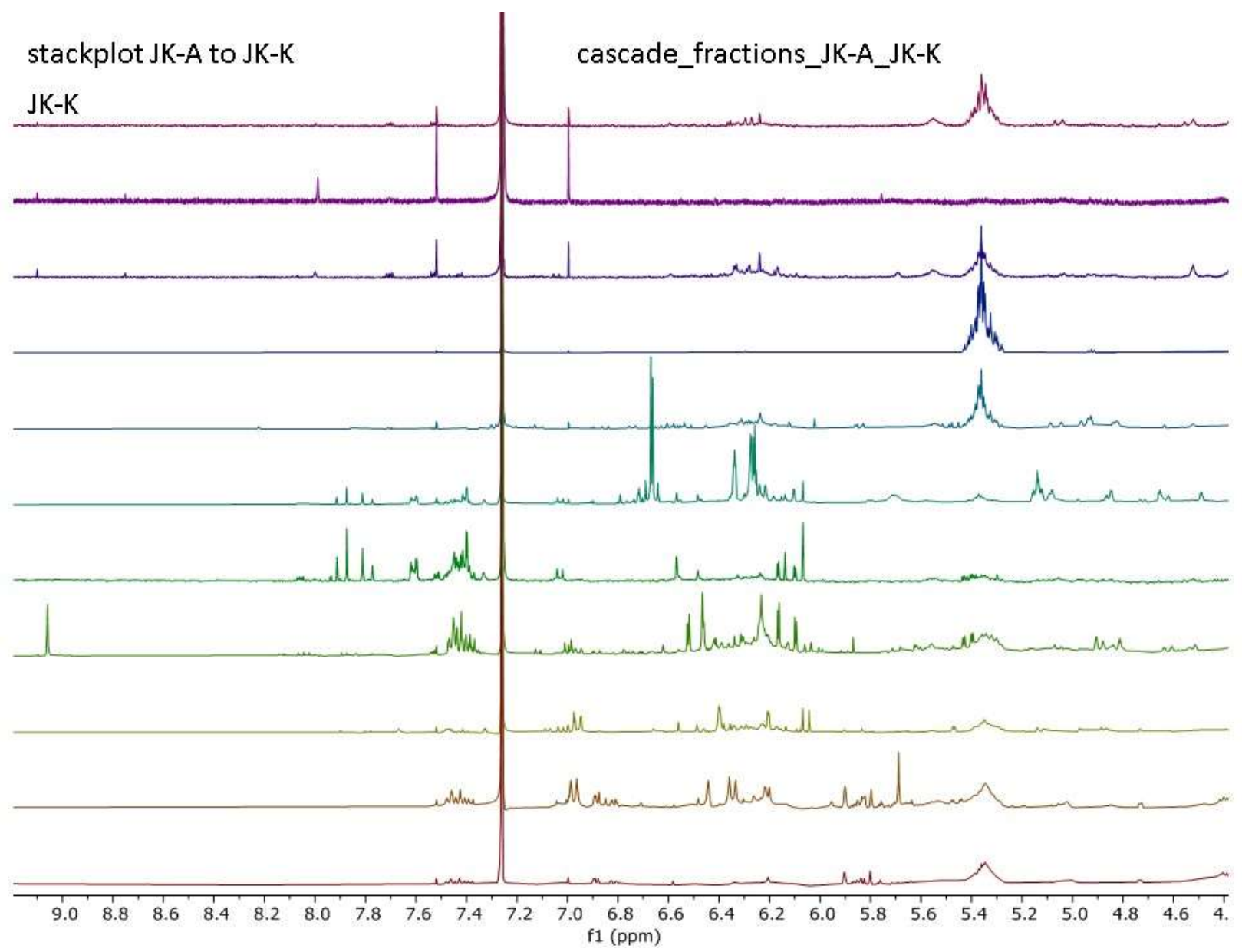


Figure S23. Low-frequency region stacked plot of ${ }^{1} \mathrm{H}$ NMR spectra (400 $\left.\mathrm{MHz}, \mathrm{CDCl}_{3}\right)$.

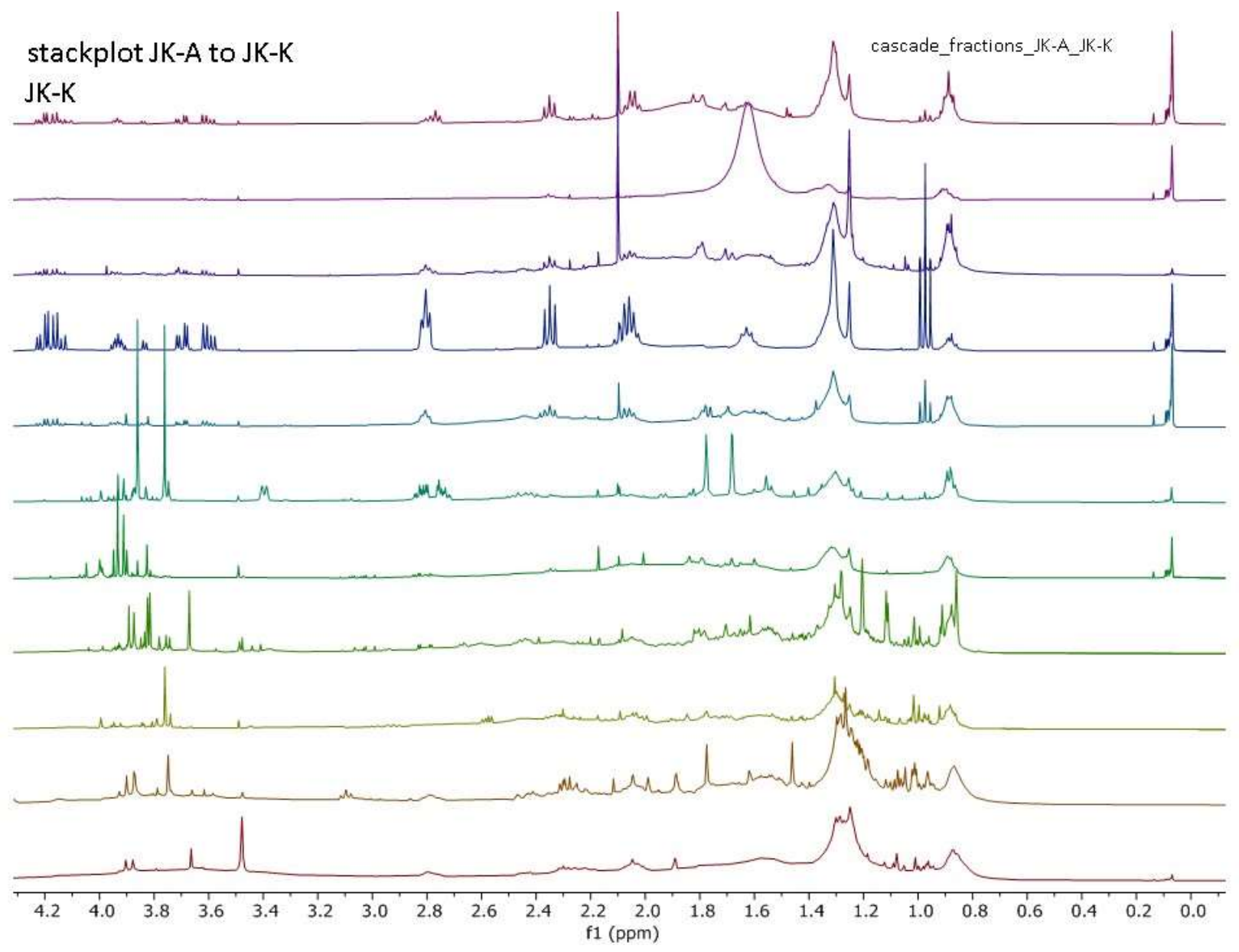


Figure S24. ${ }^{1} \mathrm{H}$ NMR Spectrum of CBN (5) in fraction C-F (400 MHz, $\left.\mathrm{CDCl}_{3}\right)$.

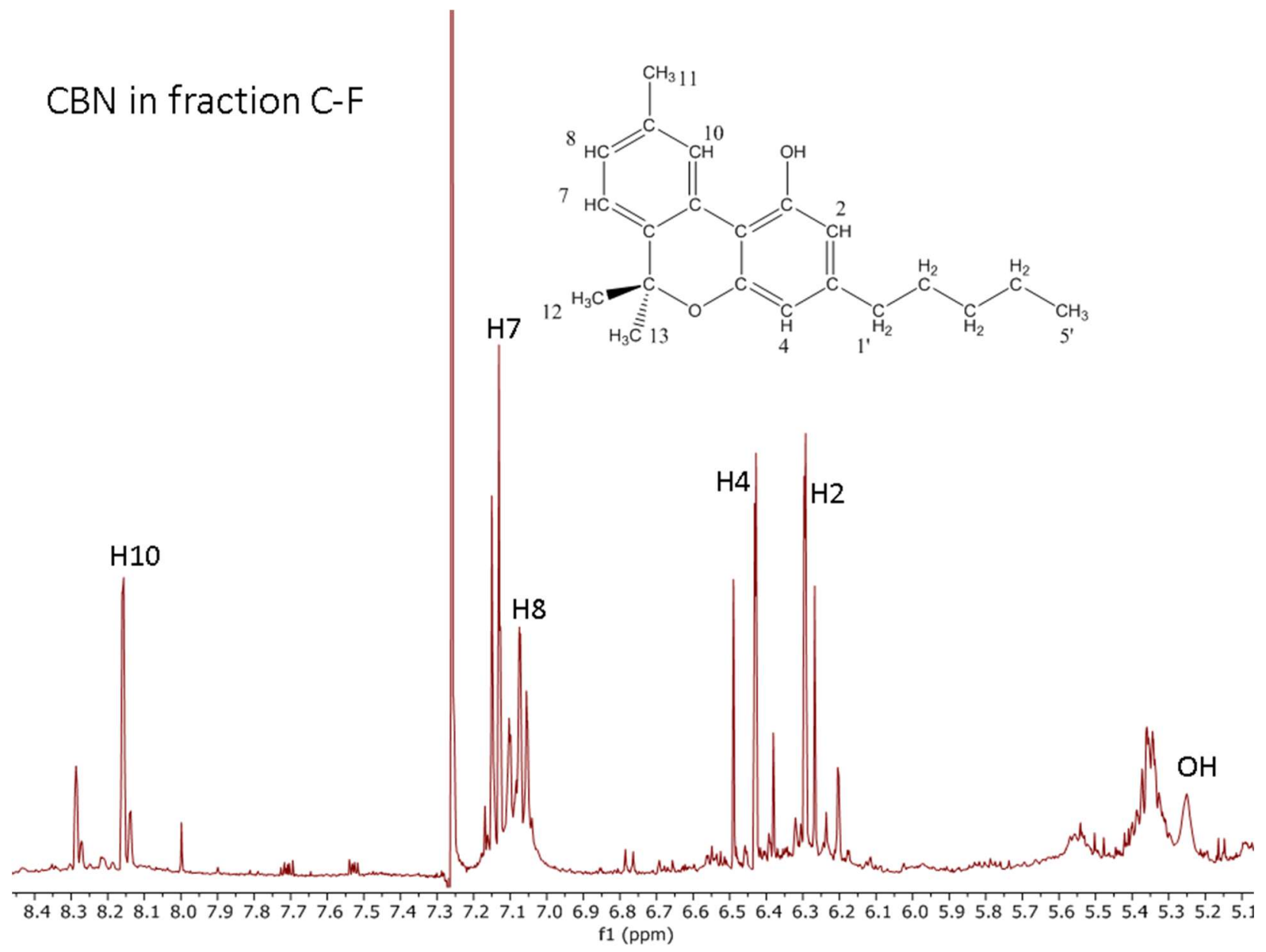


Figure S25. ${ }^{13} \mathrm{C}$ NMR Spectrum of CBD (1) $\left(100 \mathrm{MHz}, \mathrm{CDCl}_{3}\right)$.

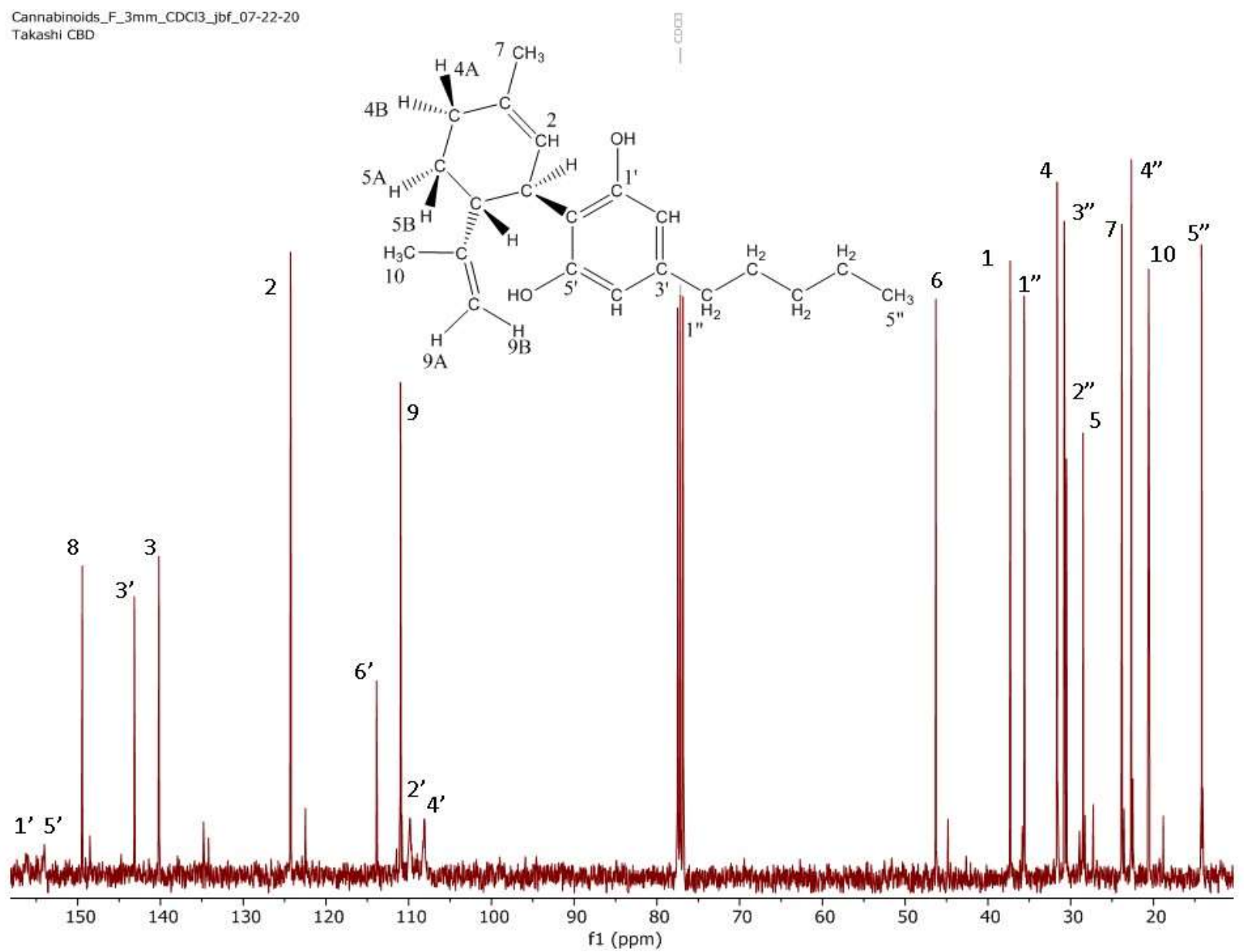


Figure S26. ${ }^{13} \mathrm{C}$ NMR Spectrum of trans $-\Delta^{9}$-THC (2a) $\left(100 \mathrm{MHz}, \mathrm{CDCl}_{3}\right)$.

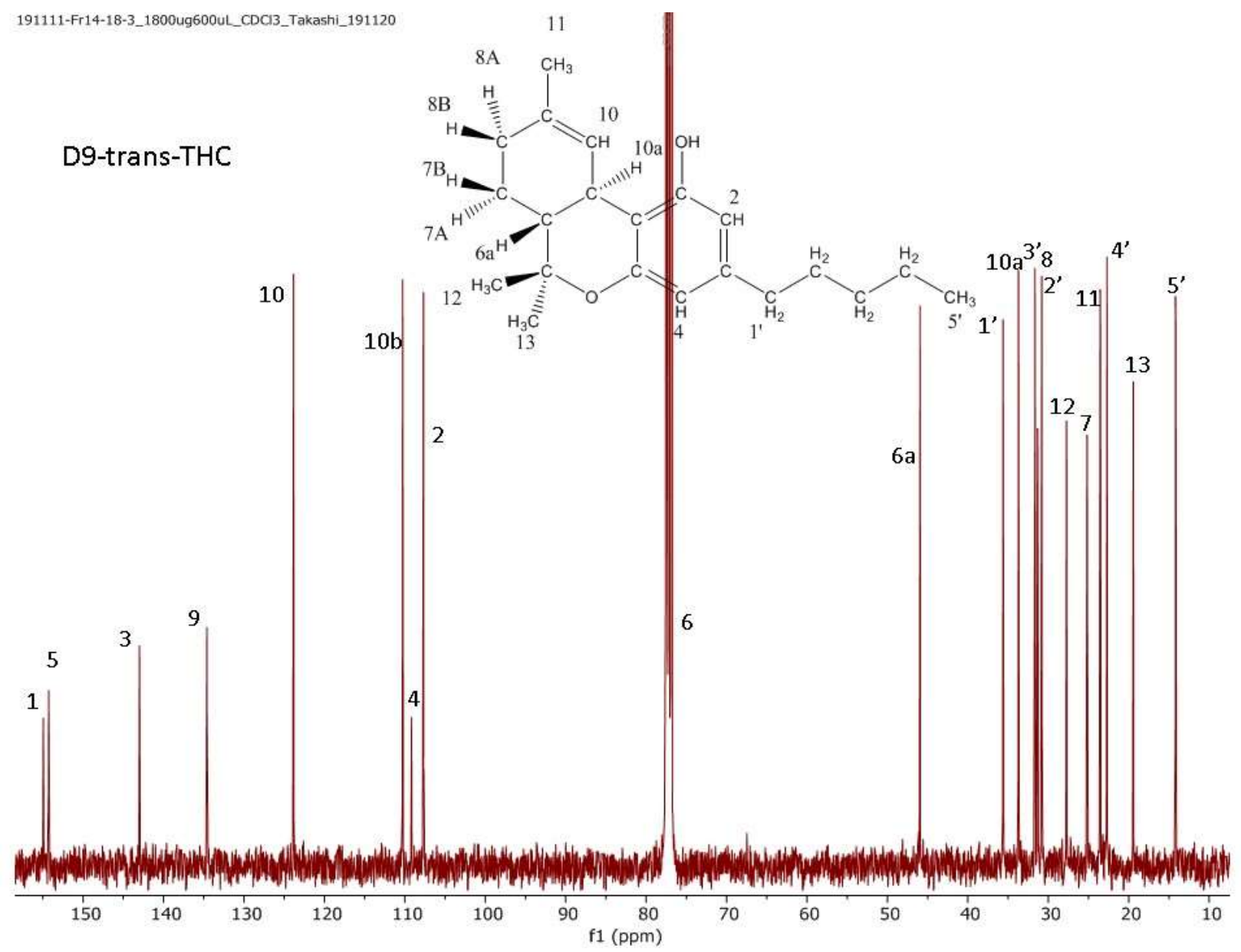


Figure S27. ${ }^{13} \mathrm{C}$ NMR Spectrum of $c i s-\Delta^{9}-\mathrm{THC}(\mathbf{2 b})\left(100 \mathrm{MHz}, \mathrm{CDCl}_{3}\right)$.

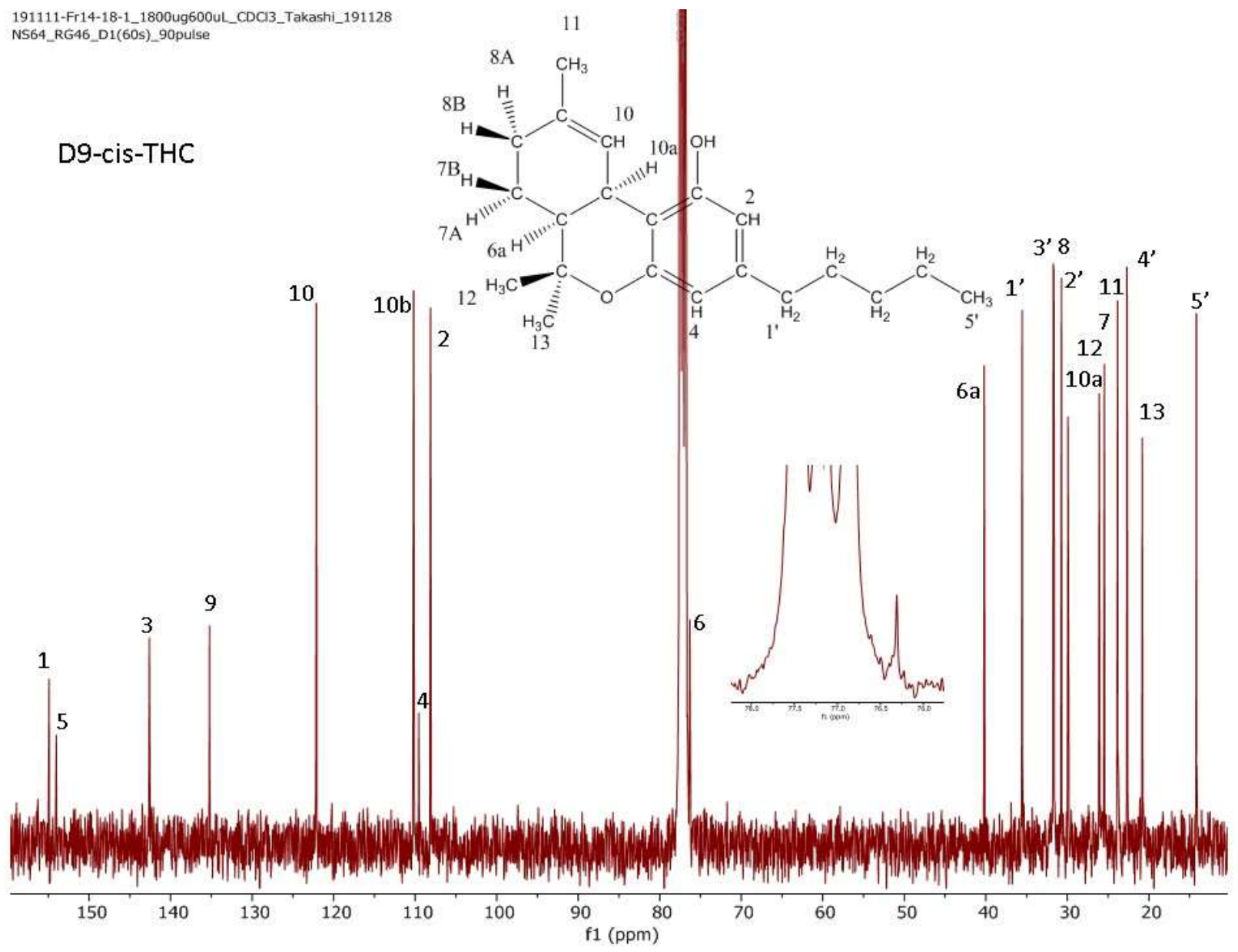


Figure S28. ${ }^{13} \mathrm{C}$ NMR Spectrum of CBC (3) $\left(100 \mathrm{MHz}, \mathrm{CDCl}_{3}\right)$.

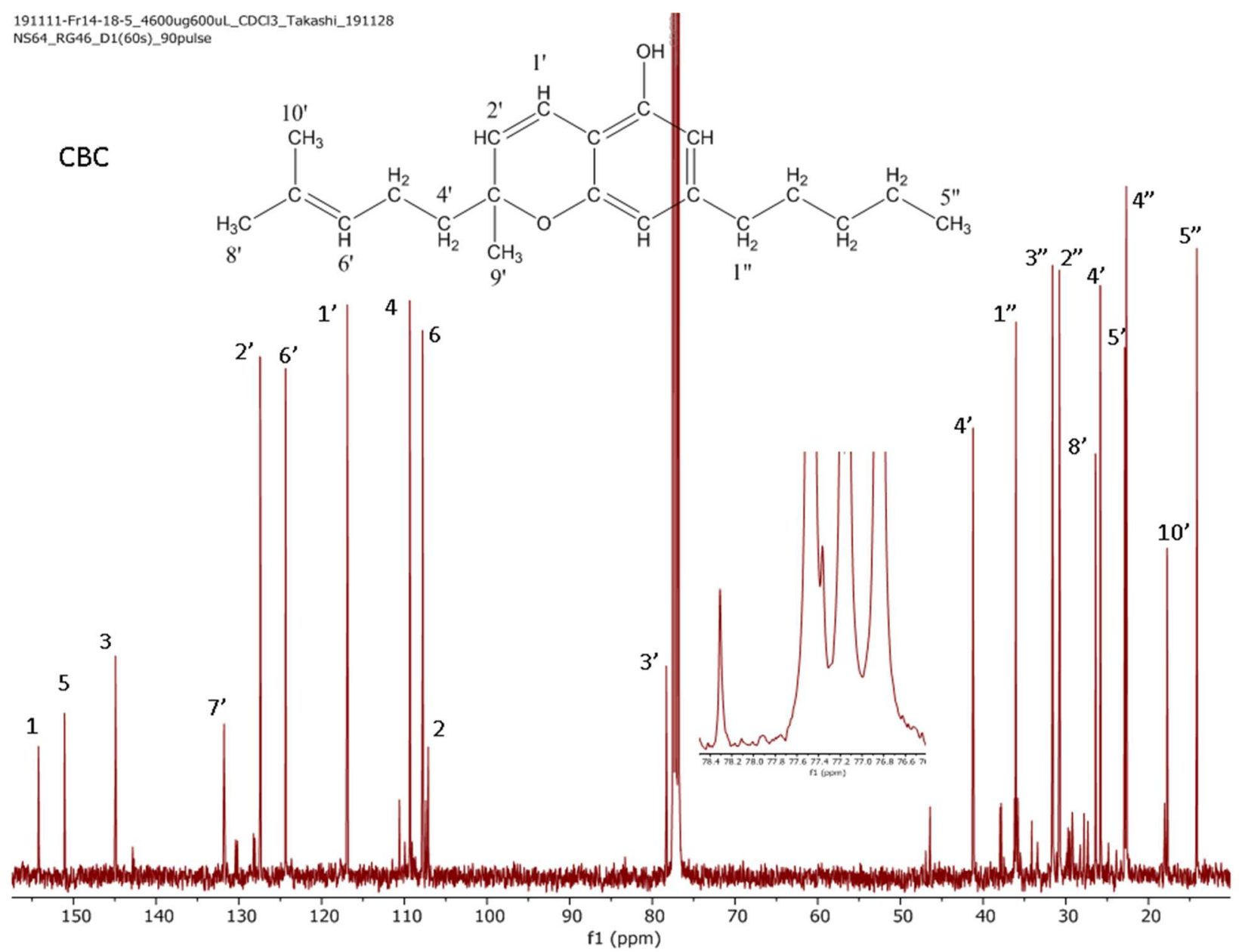


Figure S29. ${ }^{13} \mathrm{C}$ NMR Spectrum of CBG (4) $\left(100 \mathrm{MHz}, \mathrm{CDCl}_{3}\right)$.

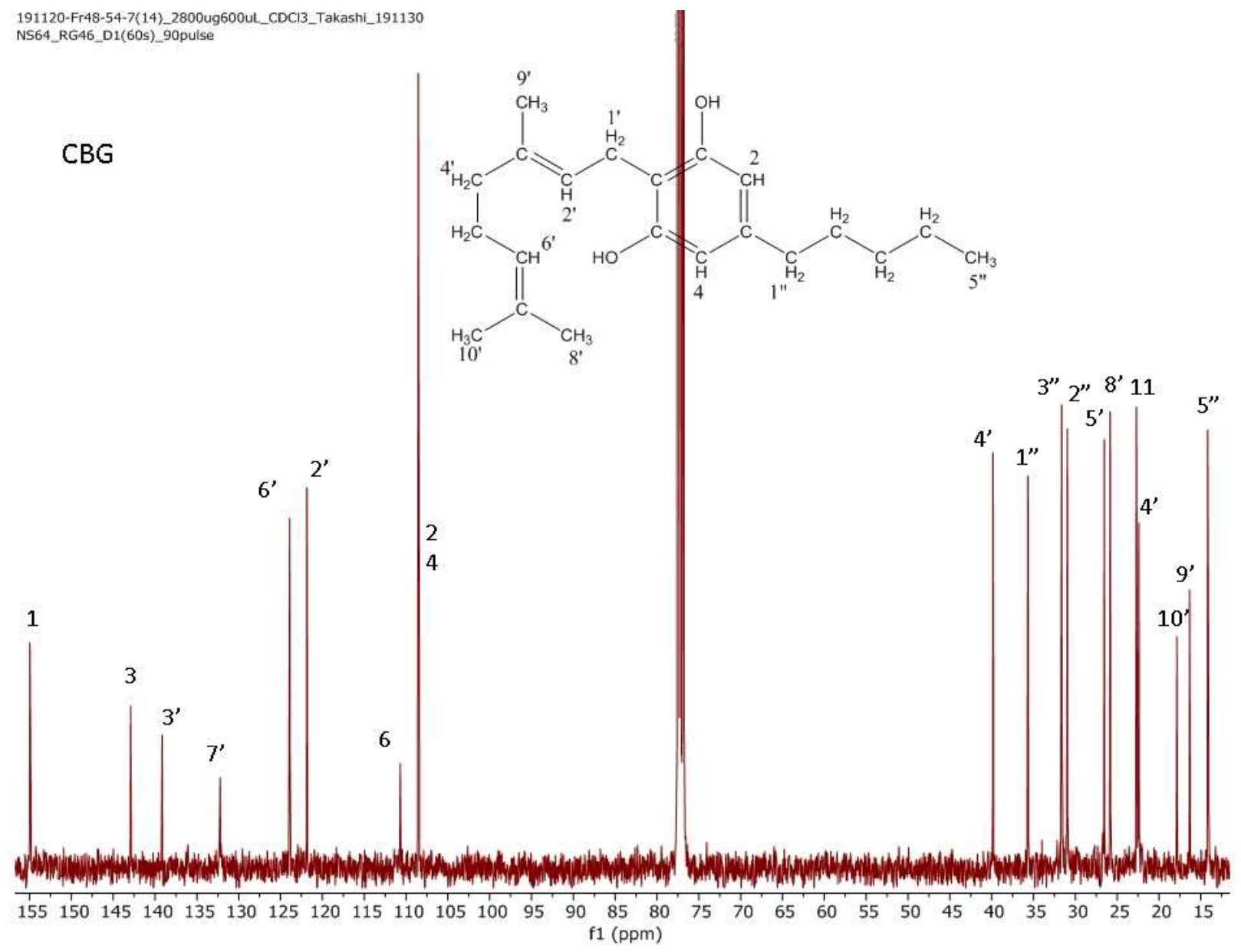


Figure S30. ${ }^{13} \mathrm{C}$ NMR Spectrum of CBN (5) $\left(100 \mathrm{MHz}, \mathrm{CDCl}_{3}\right)$.

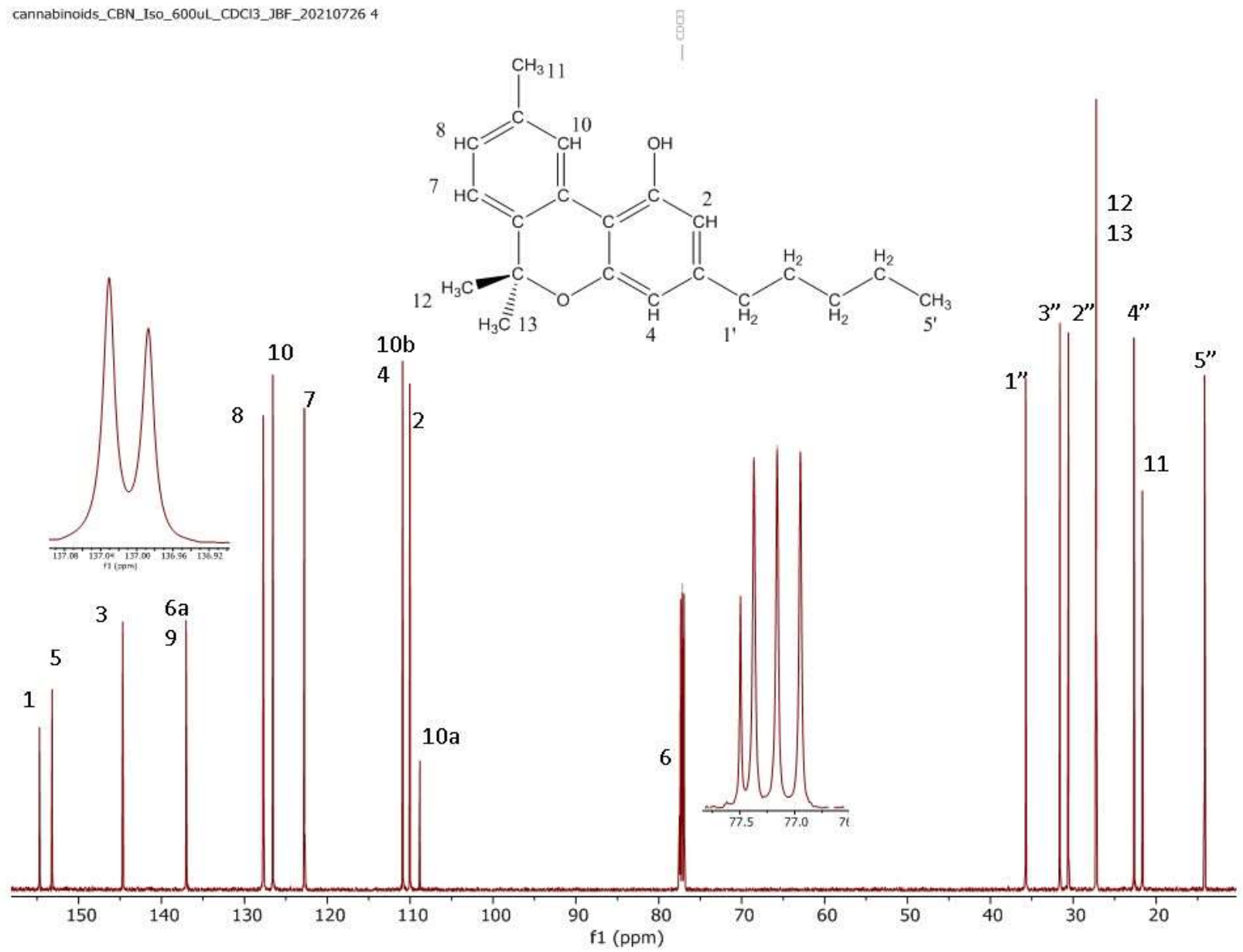


Figure S31. ${ }^{13} \mathrm{C}$ NMR Spectrum of CBDV (6) $\left(100 \mathrm{MHz}, \mathrm{CDCl}_{3}\right)$

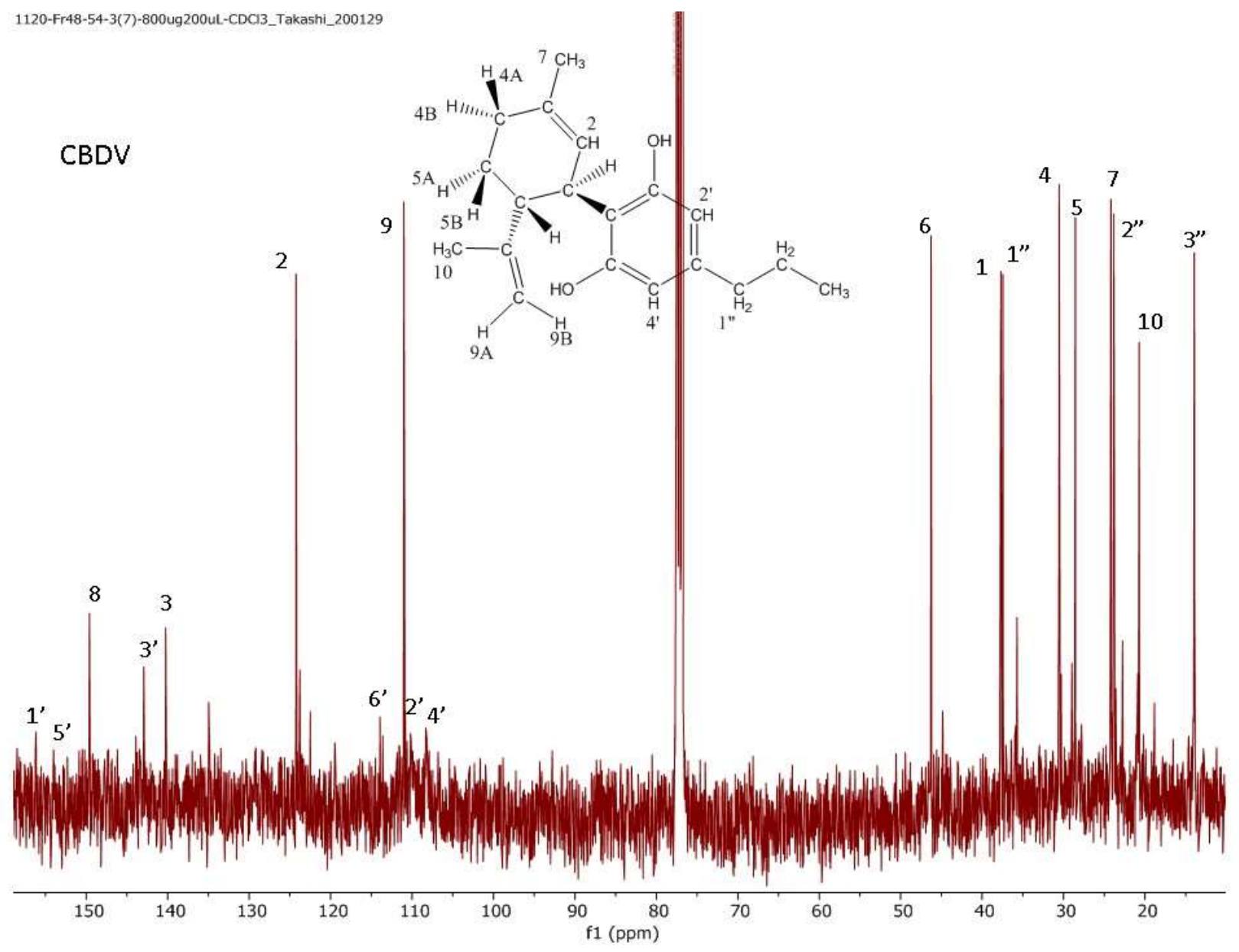


Figure S32. Calibration Curve for $\mathrm{EC} \mathrm{DMSO}_{2}$.

\begin{tabular}{lllllll} 
Solvent & Class & Tube & File Name & $\mathrm{mM}$ & $\begin{array}{l}\text { absolute } \\
\text { integral value }\end{array}$ & 90pw \\
\hline & EC1 & & DMSO2_C_5mm_1659u3300uL & 5.335 & 0.741 & 7.298 \\
CDCl3 & EC2 & $5 \mathrm{~mm}$ & DMSO2_C_5mm_1701u1100uL & 16.412 & 2.315 & 7.262 \\
& EC3 & & DMSO2_C_5mm_3683u1200uL & 32.573 & 4.573 & 7.278 \\
\hline
\end{tabular}

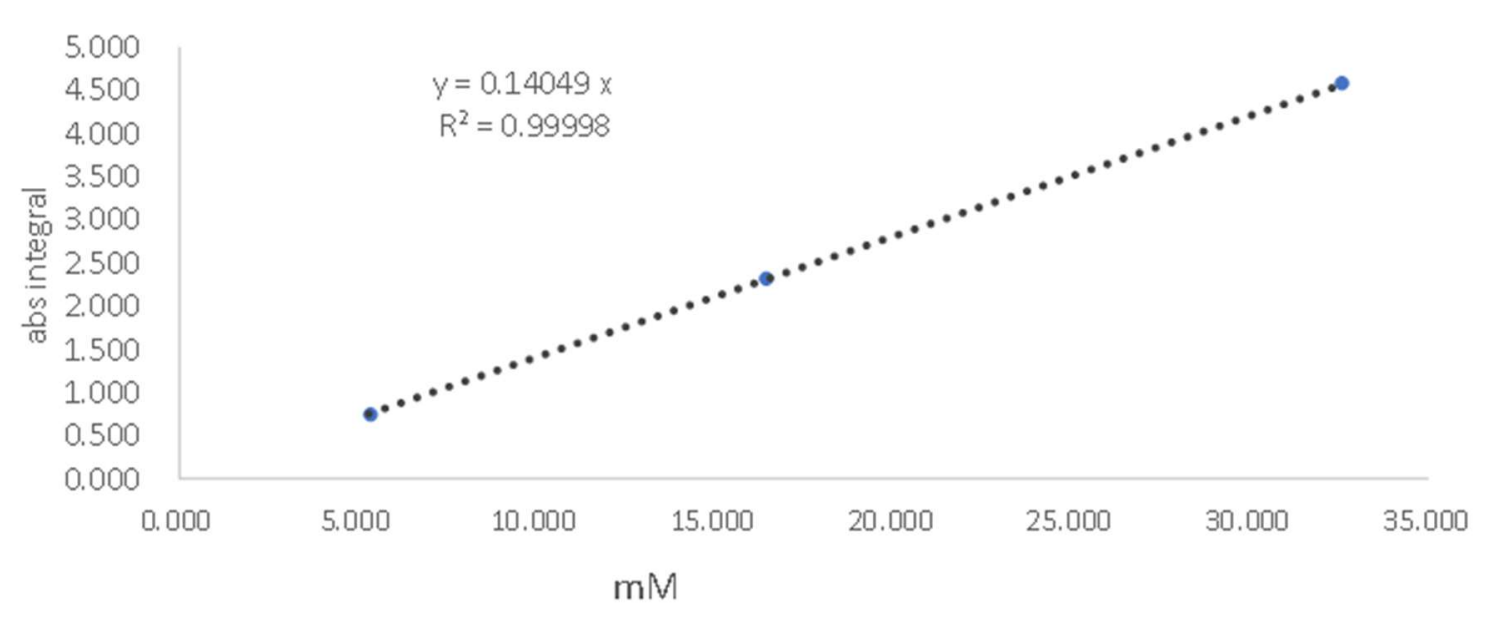


Table S33. The ${ }^{1} \mathrm{H}$ iterative full spin analysis (HiFSA) profiles of CBD (1),

\begin{tabular}{|c|c|c|c|c|c|c|c|c|}
\hline \multicolumn{9}{|c|}{ Name: $\quad$ CBD } \\
\hline \multicolumn{4}{|c|}{ CHEMKCAL SHIFTS (ppm) } & \multicolumn{5}{|c|}{ COUPLING CONSTANTS $(\mathrm{Hz})$} \\
\hline s'. жprem & $1 \mathrm{H}$ & & & 3aspen & Name & Shift & Shift & $\mathrm{Hz}$ \\
\hline cBo & $1^{\prime}(\mathrm{OH})$ & & 4.7593 & $\mathrm{CBD}$ & $H 1^{\prime} \mathrm{A}, \mathrm{H} 1^{\prime} \mathrm{E}-\mathrm{H} 2^{\prime} \mathrm{A}, \mathrm{H} \mathbf{2}^{\prime} \mathrm{B}$ & H1'A,H1'B & $\mathrm{H} Z^{\prime} \mathrm{A}, \mathrm{HZ} \mathrm{Z}^{\prime} \mathrm{B}$ & 8.65 \\
\hline CBD & $5^{\prime}(\mathrm{OH})$ & & 59969 & $\mathrm{CBD}$ & 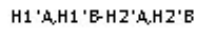 & $H 1^{\prime} A, H 11^{\prime} B$ & $\mathrm{H} Z^{\prime} \mathrm{A}, \mathrm{H} Z^{\prime} \mathrm{B}$ & 685 \\
\hline CBD & H1 & & 38582 & $\mathrm{CBD}$ & $\mathrm{H} 1-\mathrm{Hz}$ & H1 & $\mathrm{Hz}$ & 285 \\
\hline CBD & $H 1^{\prime} A, H 1^{\prime} B$ & & 2.4358 & $\mathrm{CBD}$ & H1-H6 & $\mathrm{H} 1$ & $\mathrm{H} 6$ & 10.78 \\
\hline CBD & H1O & & 1.6603 & CBD & $\mathrm{H} 1-\mathrm{H} 7$ & H1 & $\mathrm{H} 7$ & 2.49 \\
\hline CBD & $\mathrm{Hz}$ & & 55724 & $\mathrm{CBO}$ & H1O-H9A & H1O & H9A & -137 \\
\hline Cro & $H Z^{\prime} A, H Z^{\prime} B$ & & 15561 & $\mathrm{CBO}$ & H10-H9B & H1O & $\mathrm{HgB}$ & -0.63 \\
\hline CBD & $\mathrm{Hz}^{\prime}$ & & 6.2730 & CBD & 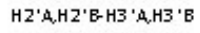 & H3 'A, H3' B & $\mathrm{H} Z^{\prime} \mathrm{A}, \mathrm{H} \mathrm{Z}^{\prime} \mathrm{B}$ & 6.01 \\
\hline CBD & $H 3^{\prime} A, H 3$ ' $B$ & & 1.2782 & $\mathrm{CBD}$ & 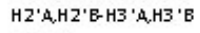 & H3' 'A, H3 'B & $\mathrm{HZ} \mathrm{Z}^{\prime} \mathrm{A}, \mathrm{H} \mathrm{Z}^{\prime} \mathrm{B}$ & 9.3 \\
\hline CBD & $\mathrm{H}_{4} \mathrm{~A}, \mathrm{H} \mathrm{H}^{\prime} \mathrm{B}$ & & 13146 & CED & $\mathrm{H} 2-\mathrm{H} 1$ & H1 & $\mathrm{Hz}^{\prime}$ & 1.00 \\
\hline CBD & $\mathrm{H}^{4}$ & & 6.1729 & $\mathrm{CBD}$ & $\mathrm{HZ}^{\prime}-\mathrm{H}^{\prime}$ & $\mathrm{H}^{\prime}$ & $\mathrm{Hz}^{\prime}$ & 3.08 \\
\hline CBD & $\mathrm{H} 4 \mathrm{~A}$ & & 2.2345 & CBD & $\mathrm{Hz}-\mathrm{H} 4 \mathrm{~A}$ & $\mathrm{~Hz}$ & $\mathrm{H} 4 \mathrm{AM}$ & -1.45 \\
\hline CBD & $\mathrm{H} 4 \mathrm{~B}$ & & 2.0987 & $\mathrm{CBO}$ & $\mathrm{Hz}-\mathrm{H} 4 \mathrm{~B}$ & $\mathrm{~Hz}$ & $\mathrm{H} 4 \mathrm{~B}$ & 135 \\
\hline CBD & H5 ' & & 08799 & CED & $\mathrm{Hz}-\mathrm{H} 7$ & $\mathrm{~Hz}$ & $\mathrm{H} 7$ & -1.12 \\
\hline CBD & H5a & & 1.7848 & CBD & $H 3^{\prime} A, H 3^{\prime} E-H_{4}{ }^{\prime} A, H_{4}{ }^{\prime} B$ & $\mathrm{H}_{4}{ }^{\prime} \mathrm{A}, \mathrm{H} 4^{\prime} \mathrm{B}$ & H3 'A, H3 'B & 953 \\
\hline CBD & H5B & & 18291 & $\mathrm{CBD}$ & $\mathrm{H} 3^{\prime} \mathrm{A}, \mathrm{H} 3^{\prime} \mathrm{E}-\mathrm{H}_{4} \mathrm{~A}, \mathrm{H}_{4} \mathrm{C}^{\prime} \mathrm{B}$ & $H_{4}^{\prime} A, H_{4}{ }^{\prime} B$ & H3 'A, H3 'B & 6.06 \\
\hline CBD & H6 & & 2.4026 & CBD & $\mathrm{H}^{\prime}{ }^{\prime A, H} \mathbf{H}^{\prime} \mathrm{E}-\mathrm{HS}$ ' $^{\prime}$ & H5 ' & $\mathrm{H}_{4}{ }^{\prime} \mathrm{A}, \mathrm{H} 4^{\prime} \mathrm{B}$ & 734 \\
\hline CBD & $\mathrm{H} 7$ & & 1.7936 & CBD & $\mathrm{H} 4:-\mathrm{H} 1$ & H1 & $\mathrm{H}^{\prime}$ & 100 \\
\hline CBD & $\mathrm{HgA}$ & & 4.6589 & CBD & $\mathrm{H} 4 \mathrm{~A}-\mathrm{H} 5 \mathrm{~A}$ & $\mathrm{H} 4 \mathrm{AM}$ & $\mathrm{H} 5 \mathrm{~A}$ & 1136 \\
\hline CBD & $\mathrm{HgB}$ & & 45573 & $\mathrm{CBD}$ & $\mathrm{H} 4 \mathrm{4}-\mathrm{H} 5 \mathrm{~B}$ & $\mathrm{H} 4.4 \mathrm{~A}$ & $\mathrm{H} 5 \mathrm{~B}$ & 5.21 \\
\hline \multirow[t]{3}{*}{$\mathrm{H} 2 \mathrm{O}$} & $\mathrm{H} 2 \mathrm{O}$ & & 1.7274 & $\mathrm{CBO}$ & $\mathrm{H} 44 \mathrm{4}-\mathrm{H} 7$ & $\mathrm{H} 4 . \mathrm{A}$ & $\mathrm{H} 7$ & -184 \\
\hline & & & & CED & $\mathrm{H} 4 \mathrm{E}-\mathrm{H} 4 \mathrm{AM}$ & $\mathrm{H} 4 \mathrm{~B}$ & $\mathrm{H} 4 \mathrm{AM}$ & -17.75 \\
\hline & $13 \mathrm{C}$ & \multicolumn{2}{|c|}{ ppm } & CBD & $\mathrm{H} 4 \mathrm{E}-\mathrm{H} 5 \mathrm{~A}$ & $\mathrm{H} 4 \mathrm{~B}$ & $\mathrm{H} 5 \mathrm{~A}$ & 494 \\
\hline CBO & & $1^{\prime}$ & 156.1906 & CED & H4E-H5B & $\mathrm{H} 4 \mathrm{~B}$ & $\mathrm{H} 5 \mathrm{~B}$ & 2.12 \\
\hline CBD & & $5^{\prime}$ & 154.1038 & CBD & $\mathrm{H} 4 \mathrm{E}-\mathrm{H} 7$ & $\mathrm{H} 4 \mathrm{~B}$ & $\mathrm{H} 7$ & -1.8 \\
\hline CBD & & 8 & 149.4383 & $\mathrm{CBO}$ & H5A-H5B & $\mathrm{H} 5 \mathrm{~A}$ & H5B & -1288 \\
\hline CBD & & 3 & 143.1579 & CBD & $\mathrm{H} 6-\mathrm{H} 1 \mathrm{O}$ & H6 & H1O & 0,00 \\
\hline CBD & & 3 & 140.1739 & CBD & $\mathrm{H} 6-\mathrm{H} 2$ & $\mathrm{H} 6$ & $\mathrm{HZ}$ & 0,0 \\
\hline CBD & & 2 & 124.599 & $\mathrm{CBD}$ & H6-H5 A & H6 & H5A & 1235 \\
\hline CBD & & $6^{\prime}$ & 1138857 & $\mathrm{CBO}$ & $\mathrm{H} 6-\mathrm{H} 5 \mathrm{~B}$ & H6 & $\mathrm{H} 5 \mathrm{~B}$ & 2.79 \\
\hline CBD & & 9 & 1109821 & $\mathrm{CBO}$ & $\mathrm{H6}-\mathrm{HgA}$ & H6 & $\mathrm{HgA}$ & -0.04 \\
\hline CBD & & $z^{\prime}$ & 109.7716 & $\mathrm{CBD}$ & H6- $\mathrm{H9B}$ & H6 & H9B & -0.70 \\
\hline CBD & & 4 & 108.0658 & $\mathrm{CBD}$ & $\mathrm{H9H}-\mathrm{HgB}$ & $\mathrm{HgA}$ & $\mathrm{HgB}$ & 2.13 \\
\hline CBD & & 6 & 46.384 & & & & & \\
\hline CED & & 1 & 37.2992 & & & & & \\
\hline CBD & & $1^{\prime}$ & $3560 \mathrm{Z}$ & & & & & \\
\hline CED & & 4 & 315024 & & & & & \\
\hline CBD & & $3^{\prime}$ & 30.7663 & & & & & \\
\hline CBD & & $2^{\prime}$ & 305776 & & & & & \\
\hline CBD & & 5 & $\mathbb{8} 5077$ & & & & & \\
\hline CBD & & 7 & 258064 & & & & & \\
\hline CBD & & $4^{\prime}$ & 22.665 & & & & & \\
\hline CBD & & 10 & 205676 & & & & & \\
\hline CBD & & $5^{\prime}$ & 14.1657 & & & & & \\
\hline
\end{tabular}


Table 34. The ${ }^{1} \mathrm{H}$ iterative full spin analysis (HiFSA) profiles of trans $-\Delta^{9}-\mathrm{THC}(\mathbf{2 b})$.

\begin{tabular}{|c|c|c|c|c|c|c|c|}
\hline \multicolumn{8}{|l|}{ Name: } \\
\hline \multicolumn{4}{|c|}{ CHEMICALSHIFTS (ppm) } & \multicolumn{4}{|c|}{ COUPLING CONSTANTS ( $\mathrm{Hz})$} \\
\hline Spircystem m & Name & $\mathrm{ppm}$ & Spirzystrm m & Name & Shift & Shift & $\mathrm{Hz}$ \\
\hline t-D9-THC & $1(\mathrm{OH})$ & 4.6954 & t-D9-THC & $\mathrm{H}^{\prime}{ }^{\prime} \mathrm{A}, \mathrm{H} 1^{\prime} \mathrm{B}-\mathrm{H} 2^{\prime} \mathrm{A}, \mathrm{H} 2^{\prime} \mathrm{B}$ & $\mathrm{H} 2^{\prime} \mathrm{A}, \mathrm{H} 2^{\prime} \mathrm{B}$ & H1'A, H1'B & 7.81 \\
\hline t-D9-THC & H1'A, H1'B & 2.4345 & t-D9-THC & $\mathrm{H} 10-\mathrm{H} 10 \mathrm{a}$ & $\mathrm{H} 10$ & $\mathrm{H} 10 \mathrm{a}$ & 2.63 \\
\hline t-D9-THC & $\mathrm{H} 10$ & 6.2963 & t-D9-THC & $\mathrm{H} 10-\mathrm{H} 11(\mathrm{Me})$ & $\mathrm{H} 10$ & H11(Me) & -1.40 \\
\hline t-D9-THC & $\mathrm{H} 10 \mathrm{a}$ & 3.1976 & t-D9-THC & $\mathrm{H} 10-\mathrm{H} 6 \mathrm{a}$ & $\mathrm{H} 10$ & $\mathrm{H} 6 \mathrm{a}$ & 0.60 \\
\hline t-D9-THC & H11(Me) & 1.6794 & t-D9-THC & H10-H8A & H1O & H8A. & -0.36 \\
\hline t-D9-THC & $\mathrm{H} 12(\mathrm{Me})$ & 1.4095 & t-D9-THC & H10-H8B & $\mathrm{H} 10$ & $\mathrm{H} 8 \mathrm{~B}$ & 0.38 \\
\hline t-D9-THC & H13(Me) & 1.0908 & t-D9-THC & $\mathrm{H} 10 \mathrm{a}-\mathrm{H} 1 \mathrm{l}(\mathrm{Me})$ & $\mathrm{H} 10 \mathrm{a}$ & H11(Me) & 2.21 \\
\hline t-D9-THC & $\mathrm{H}_{2}$ & 6.1383 & t-D9-THC & $\mathrm{H}^{\prime}{ }^{\prime} \mathrm{A}, \mathrm{H} 2^{\prime} \mathrm{B}-\mathrm{H} 3^{\prime} \mathrm{A}, \mathrm{H} 3^{\prime} \mathrm{B}$ & H3'A, H3'B & $\mathrm{H} 2^{\prime} \mathrm{A}, \mathrm{H} 2^{\prime} \mathrm{B}$ & 7.39 \\
\hline t-D9-THC & $\mathrm{H} 2^{\prime} \mathrm{A}, \mathrm{H} 2^{\prime} \mathrm{B}$ & 1.5567 & t-D9-THC & $\mathrm{H} 2-\mathrm{H} 10 \mathrm{a}$ & $\mathrm{H}_{2}$ & $\mathrm{H} 10 \mathrm{a}$ & 0.31 \\
\hline t-D9-THC & H3'A, H3'B & 1.3004 & $t-D 9-T H C$ & $\mathrm{H} 2-\mathrm{H}_{4}$ & $\mathrm{H} 2$ & $\mathrm{H} 4$ & 0.65 \\
\hline t-D9-THC & $\mathrm{H} 4$ & 6.2687 & t-D9-THC & $\mathrm{H} 2-\mathrm{H} 6 \mathrm{a}$ & $\mathrm{H} 2$ & $\mathrm{H} 6 \mathrm{a}$ & -1.19 \\
\hline t-D9-THC & $\mathrm{H} 4^{\prime} \mathrm{A}, \mathrm{H} 4^{\prime} \mathrm{B}$ & 1.2980 & t-D9-THC & $\mathrm{H} 22 \mathrm{~A}-\mathrm{H} 22 \mathrm{~B}$ & $\mathrm{H}^{\prime} \mathrm{A}, \mathrm{H} 4^{\prime} \mathrm{B}$ & $\mathrm{H} 4^{\prime} \mathrm{A}, \mathrm{H} 4^{\prime} \mathrm{B}$ & -12.84 \\
\hline t-D9-THC & H5' & 0.8777 & t-D9-THC & $\mathrm{H}^{\prime} \mathrm{A}, \mathrm{H}^{\prime} \mathrm{B}-\mathrm{B}-\mathrm{H}^{\prime} \mathrm{A}, \mathrm{H} 4^{\prime} \mathrm{B}$ & $\mathrm{H}^{\prime} \mathrm{A}, \mathrm{H} 4^{\prime} \mathrm{B}$ & H3'A, H3'B & 8.04 \\
\hline t-D9-THC & $\mathrm{H} 6 \mathrm{a}$ & 1.6904 & t-D9-THC & H3'A, H3'B-H5' & H5' & $\mathrm{H} 3^{\prime} \mathrm{A}, \mathrm{H} 3^{\prime} \mathrm{B}$ & -0.67 \\
\hline $\mathrm{t}-\mathrm{Dg}$-THC & $H 7 A$ & 1.9140 & t-D9-THC & $\mathrm{H} 4-\mathrm{H} 10 \mathrm{a}$ & $\mathrm{H}_{4}$ & $\mathrm{H} 10 \mathrm{a}$ & 0.32 \\
\hline t-D9-THC & H7B & 1.4051 & t-D9-THC & $\mathrm{H} 4 \mathrm{H} 6 \mathrm{a}$ & $\mathrm{H} 4$ & $\mathrm{H} 6 \mathrm{a}$ & -1.16 \\
\hline t-D9-THC & $\mathrm{H} 8 \mathrm{~A}$ & 2.1624 & t-D9-THC & $\mathrm{H} 5^{\prime}-\mathrm{H} 22 \mathrm{~A}$ & H5' & $\mathrm{H} 4^{\prime} \mathrm{A}, \mathrm{H} 4^{\prime} \mathrm{B}$ & 7.47 \\
\hline \multirow[t]{3}{*}{ t-D9-THC } & $\mathrm{H} 8 \mathrm{~B}$ & 2.1677 & t-D9-THC & $\mathrm{H} 6 a-\mathrm{H} 10 \mathrm{a}$ & $\mathrm{H} 6 \mathrm{a}$ & $\mathrm{H} 10 \mathrm{a}$ & 10.99 \\
\hline & & & t-D9-THC & H6a-H12(Me) & $\mathrm{H} 6 \mathrm{a}$ & H12(Me) & -0.24 \\
\hline & $13 \mathrm{C}$ & ppm & t-D9-THC & H6a-H13(Me) & $\mathrm{H} 6 \mathrm{a}$ & H13(Me) & -0.14 \\
\hline t-D9-THC & 1 & 1549373 & t-D9-THC & $\mathrm{H} 6 \mathrm{a}-\mathrm{H} 7 \mathrm{~A}$ & $\mathrm{H} 6 \mathrm{a}$ & $H 7 A$ & 2.12 \\
\hline t-D9-THC & 5 & 154.2775 & t-D9-THC & H6a-H7B & $\mathrm{H} 6 \mathrm{a}$ & $\mathrm{H} 7 \mathrm{~B}$ & 12.49 \\
\hline t-D9-THC & 3 & 142.9864 & t-D9-THC & $\mathrm{H} 7 \mathrm{~A}_{-} \mathrm{H} 7 \mathrm{~B}$ & H7A & $\mathrm{H} 7 \mathrm{~B}$ & -12.72 \\
\hline t-D9-THC & 9 & 134.6058 & t-D9-THC & H7A-H8A & $\mathrm{H} 7 \mathrm{~A}$ & $\mathrm{H} 8 \mathrm{~A}$ & 3.96 \\
\hline t-D9-THC & 10 & 123.833 & t-D9-THC & H7A-H8B & $H 7 A$ & $\mathrm{H} 8 \mathrm{~B}$ & 3.87 \\
\hline t-D9-THC & $10 \mathrm{~b}$ & 110.2634 & t-D9-THC & H7E-H8A & $\mathrm{H} 7 \mathrm{~B}$ & $\mathrm{H} 8 \mathrm{~A}$ & 12.11 \\
\hline t-D9-THC & 4 & 109.1607 & t-D9-THC & H7B-H8B & $\mathrm{H} 7 \mathrm{~B}$ & $\mathrm{H} 8 \mathrm{~B}$ & 5.70 \\
\hline t-D9-THC & 2 & 107.6692 & t-D9-THC & $\mathrm{H} 8 \mathrm{~A}-\mathrm{H} 10 \mathrm{a}$ & $\mathrm{H} 8 \mathrm{~A}$ & $\mathrm{H} 10 \mathrm{a}$ & 2.43 \\
\hline t-D9-THC & 6 & 76.5461 & t-D9-THC & H8A-H11(Me) & H8A & H11(Me) & -0.04 \\
\hline $\mathrm{t}$-D9-THC & $6 a$ & 45.9371 & $\mathrm{t}-\mathrm{D} 9-\mathrm{THC}$ & $\mathrm{H} 8 \mathrm{~A}-\mathrm{H} 8 \mathrm{~B}$ & H8A & $\mathrm{H} 8 \mathrm{~B}$ & -14.53 \\
\hline t-D9-THC & $1^{\prime}$ & 35.6163 & t-DG-THC & H8E-H10a & H8B & $\mathrm{H} 10 \mathrm{a}$ & 2.68 \\
\hline t-D9-THC & $10 a$ & 33.7 & t-D9-THC & H8E-H11(Me) & $\mathrm{H} 8 \mathrm{~B}$ & H11(Me) & -1.04 \\
\hline t-D9-THC & 3 & 31.6534 & & & & & \\
\hline t-D9-THC & 8 & 31.3063 & & & & & \\
\hline t-D9-THC & $2^{\prime}$ & 30.7966 & & & & & \\
\hline t-D9-THC & 12 & 27.7188 & & & & & \\
\hline t-D9-THC & 7 & 25.1574 & & & & & \\
\hline $\mathrm{t}-\mathrm{Dg}-\mathrm{THC}$ & 11 & 23.5184 & & & & & \\
\hline t-D9-THC & $4^{\prime}$ & 22.6904 & & & & & \\
\hline t-D9-THC & 13 & 19.4168 & & & & & \\
\hline t-D9-THC & $5^{\prime}$ & 14.1605 & & & & & \\
\hline
\end{tabular}


Table S35. The ${ }^{1} \mathrm{H}$ iterative full spin analysis (HiFSA) profiles of $c i s-\Delta^{9}-\mathrm{THC}(\mathbf{2 a})$.

\begin{tabular}{|c|c|c|}
\hline \multirow{2}{*}{\multicolumn{2}{|c|}{$\begin{array}{l}\text { Name: } \quad \text { cis-D9-THo } \\
\text { CHEMICALSHIFTS (ppm) }\end{array}$}} & \multirow[b]{3}{*}{$\mathrm{ppm}$} \\
\hline & & \\
\hline Spinsys tem & Name & \\
\hline Cis-D9-THC & H1'A, H1'B & 2.4249 \\
\hline Cis-D9-THC & $\mathrm{H} 10$ & 6.2074 \\
\hline cis-D9-THC & $\mathrm{H} 10 \mathrm{a}$ & 3.5552 \\
\hline cis-D9-THC & H11(Me) & 1.6894 \\
\hline Cis-D9-THC & $\mathrm{H} 12$ (Me) & 1.3902 \\
\hline Cis-D9-THC & H13(Me) & 1.2725 \\
\hline cis-D9-THC & $\mathrm{H} 2$ & 6.1298 \\
\hline cis-D9-THC & $\mathrm{H} 2^{\prime A} \mathrm{~A}, \mathrm{H} 2^{\prime} \mathrm{B}$ & 1.5612 \\
\hline cis-D9-THC & H3'A, H3'B & 1.3421 \\
\hline Cis-D9-THC & $\mathrm{H} 4$ & 6.2463 \\
\hline cis-D9-THC & $\mathrm{H} 4^{\prime} \mathrm{A}, \mathrm{H}^{\prime} \mathrm{B}$ & 1.2888 \\
\hline cis-D9-THC & H5'(Me) & 0.8779 \\
\hline Cis-D9-THC & $\mathrm{H} 6 \mathrm{a}$ & 1.7238 \\
\hline Cis-D9-THC & $\mathrm{H} 7 \mathrm{~B}$ & 1.9287 \\
\hline cis-D9-THC & $H 7 A$ & 1.5201 \\
\hline Cis-D9-THC & $\mathrm{H} 8 \mathrm{~B}$ & 1.9943 \\
\hline Cis-D9-THC & H8A. & 1.9786 \\
\hline \multirow[t]{2}{*}{ cis-D9-THC } & $\mathrm{OH}$ & 4.7421 \\
\hline & $13 \mathrm{C}$ & $\mathrm{ppm}$ \\
\hline Cis-D9-THC & 1 & 154911 \\
\hline cis-D9-THC & 5 & 154.0197 \\
\hline cis-D9-THC & 3 & 142.6079 \\
\hline Cis-D9-THC & 9 & 135.2224 \\
\hline Cis-D9-THC & 10 & 122.1876 \\
\hline Cis-D9-THC & 4 & 110.1727 \\
\hline Cis-D9-THC & $10 \mathrm{~b}$ & 109.5531 \\
\hline Cis-D9-THC & 2 & 108.1195 \\
\hline cis-D9-THC & 6 & 76.3101 \\
\hline cis-D9-THC & $6 a$ & 40.1938 \\
\hline Cis-D9-THC & $1^{\prime}$ & 35.5383 \\
\hline Cis-D9-THC & $3^{\prime}$ & 31.7179 \\
\hline cis-D9-THC & 8 & 31.64 \\
\hline cis-D9-THC & $2^{\prime}$ & 30.7187 \\
\hline cis-D9-THC & $10 a$ & 29.9233 \\
\hline Cis-D9-THC & 12 & 26.0725 \\
\hline cis-D9-THC & 7 & 25.4625 \\
\hline Cis-D9-THC & 11 & 23.8238 \\
\hline Cis-D9-THC & $4^{\prime}$ & $22.68 \pi$ \\
\hline cis-D9-THC & 13 & 20.8053 \\
\hline cis-D9-THC & $5^{\prime}$ & 14.1596 \\
\hline
\end{tabular}

\begin{tabular}{|c|c|c|c|c|}
\hline Spinsys tem & Name & Shift & Shift & $\mathrm{Hz}$ \\
\hline cis-D9-THC & $\mathrm{H}^{\prime}{ }^{\prime} \mathrm{A}, \mathrm{H} 1^{\prime} \mathrm{B}-\mathrm{H} 2^{\prime} \mathrm{A}, \mathrm{H} 2^{\prime} \mathrm{B}$ & H1'A, H1'B & $\mathrm{H}^{\prime}{ }^{\prime} \mathrm{A}, \mathrm{H} 2^{\prime} \mathrm{B}$ & 7.86 \\
\hline cis-D9-THC & $\mathrm{H} 10-\mathrm{H} 10 \mathrm{a}$ & $\mathrm{H} 10$ & $\mathrm{H} 10 \mathrm{a}$ & 4.91 \\
\hline cis-D9-THC & H10-H6a & $\mathrm{H} 10$ & $\mathrm{H} 6 \mathrm{a}$ & 1.60 \\
\hline cis-D9-THC & H10-H8A & $\mathrm{H} 10$ & $\mathrm{H} 8 \mathrm{~B}$ & -1.56 \\
\hline cis-D9-THC & H11(Me)-H10 & $\mathrm{H} 10$ & H11(Me) & -1.23 \\
\hline cis-D9-THC & $\mathrm{H} 11(\mathrm{Me})-\mathrm{H} 10 \mathrm{a}$ & H11(Me) & $\mathrm{H} 10 \mathrm{a}$ & 1.75 \\
\hline cis-D9-THC & H12(Me)-H6a & $\mathrm{H} 12$ (Me) & $\mathrm{H} 6 \mathrm{a}$ & -0.01 \\
\hline cis-D9-THC & H13(Me)-H6a & H13(Me) & $\mathrm{H} 6 \mathrm{a}$ & 0.00 \\
\hline cis-D9-THC & $\mathrm{H}^{\prime}{ }^{\prime A}, \mathrm{H} 2^{\prime} \mathrm{B}-\mathrm{H} 3^{\prime} \mathrm{A}, \mathrm{H} 3^{\prime} \mathrm{B}$ & $\mathrm{H}^{\prime} \mathrm{A}, \mathrm{H} 2^{\prime} \mathrm{B}$ & $\mathrm{H} 3{ }^{\prime} \mathrm{A}, \mathrm{H} 3{ }^{\prime} \mathrm{B}$ & 12.17 \\
\hline cis-D9-THC & $\mathrm{H} 2-\mathrm{H} 10 \mathrm{a}$ & $\mathrm{H} 2$ & $\mathrm{H} 10 \mathrm{a}$ & 0.01 \\
\hline cis-D9-THC & $\mathrm{H} 2-\mathrm{H}_{4}$ & $\mathrm{H} 2$ & $\mathrm{H} 4$ & 1.62 \\
\hline cis-D9-THC & $\mathrm{H}^{\prime} \mathrm{A}, \mathrm{H} 3^{\prime} \mathrm{B}-\mathrm{H} 4{ }^{\prime} \mathrm{A}, \mathrm{H} 4^{\prime} \mathrm{B}$ & H3'A, H3'B & $\mathrm{H}^{\prime} \mathrm{A}, \mathrm{H} 4^{\prime} \mathrm{B}$ & 10.29 \\
\hline cis-D9-THC & $\mathrm{H}^{\prime} \mathrm{A}, \mathrm{H} 4^{\prime} \mathrm{B}-\mathrm{H} 5^{\prime}(\mathrm{Me})$ & H5'(Me) & $\mathrm{H}_{4}^{\prime} \mathrm{A}, \mathrm{H} 4{ }^{\prime} \mathrm{B}$ & 6.89 \\
\hline cis-D9-THC & $\mathrm{H} 4 \mathrm{H} 10 \mathrm{a}$ & $\mathrm{H} 4$ & $\mathrm{H} 10 \mathrm{a}$ & 0.01 \\
\hline cis-D9-THC & $\mathrm{H} 6 \mathrm{a}-\mathrm{H} 10 \mathrm{a}$ & $\mathrm{H} 6 \mathrm{a}$ & $\mathrm{H} 10 \mathrm{a}$ & 5.62 \\
\hline cis-D9-THC & H7E-H10a & $\mathrm{H} 7 \mathrm{~B}$ & $\mathrm{H} 10 \mathrm{a}$ & 1.18 \\
\hline Cis-D9-THC & H7E-H6a & $\mathrm{H} 7 \mathrm{~B}$ & $\mathrm{H} 6 \mathrm{a}$ & 2.59 \\
\hline cis-D9-THC & $\mathrm{H} 7 \mathrm{~A}-\mathrm{H} 7 \mathrm{~B}$ & $\mathrm{H} 7 \mathrm{~B}$ & $H 7 A$ & -13.89 \\
\hline cis-D9-THC & $\mathrm{H} 7 \mathrm{~A}-\mathrm{H} 6 \mathrm{a}$ & $\mathrm{H} 7 \mathrm{~A}$ & $\mathrm{H} 6 \mathrm{a}$ & 10.56 \\
\hline Cis-D9-THC & H8B-H11(Me) & $\mathrm{H} 8 \mathrm{~B}$ & H11(Me) & 0.00 \\
\hline cis-D9-THC & H8E-H7B & $\mathrm{H} 8 \mathrm{~B}$ & $\mathrm{H} 7 \mathrm{~B}$ & 6.88 \\
\hline cis-D9-THC & H8E-H7A & $\mathrm{H} 8 \mathrm{~B}$ & $\mathrm{H} 7 \mathrm{~A}$ & 17.13 \\
\hline cis-D9-THC & $\mathrm{H} 8 \mathrm{~A}-\mathrm{H} 8 \mathrm{~B}$ & $\mathrm{H} 8 \mathrm{~A}$ & $\mathrm{H} 8 \mathrm{~B}$ & -14.35 \\
\hline cis-D9-THC & H8A-H11(Me) & $\mathrm{H} 8 \mathrm{~A}$ & H11(Me) & -1.47 \\
\hline cis-D9-THC & $\mathrm{H} 8 \mathrm{~A}-\mathrm{H} 7 \mathrm{~B}$ & H8A & $\mathrm{H} 7 \mathrm{~B}$ & 3.22 \\
\hline cis-D9-THC & H8A-H7A & $\mathrm{H} 8 \mathrm{~A}$ & $\mathrm{H} 7 \mathrm{~A}$ & 3.63 \\
\hline
\end{tabular}


Table S36. The ${ }^{1} \mathrm{H}$ iterative full spin analysis (HiFSA) profiles of CBC (3).

\begin{tabular}{|c|c|c|c|c|c|c|c|}
\hline Name: & $\mathrm{CEC}$ & & & & & & \\
\hline CHEMKCALS & & & COUPLING & TANTS $(\mathrm{Hz})$ & & & \\
\hline Spirzystem m & $1 \mathrm{H}$ & ppm & spireystem & Name & Shift & Shift & CouplinE $(\mathrm{Hz})$ \\
\hline $\operatorname{CEC}$ & $1(\mathrm{OH})$ & 4.6221 & $\mathrm{CEC}$ & $H 1^{\prime} A, H 1^{\prime} E-H 2^{\prime} A, H 2^{\prime} B$ & $H Z^{\prime} A, H Z^{\prime} B$ & H1'A,H1'B & 7.75 \\
\hline $\mathrm{CEC}$ & $H 1^{\prime} A, H 11^{\prime} B$ & 2.4400 & $\mathrm{CEC}$ & $\mathrm{H} 1^{\prime}-\mathrm{Hz}$ & $\mathrm{H}^{\prime}$ & $\mathrm{HZ}^{\prime}$ & 999 \\
\hline $\mathrm{CEC}$ & $\mathrm{H}^{\prime}$ & 6.6080 & $\mathrm{CEC}$ & HE'(Me)-H6' & Hs'(Me) & H6' & -093 \\
\hline $\mathrm{CEC}$ & HE' '(Me) & 1.6596 & $\mathrm{CEC}$ & $\mathrm{H} 2^{\prime} \mathrm{A}, \mathrm{H} \mathbf{2}^{\prime} \mathrm{E}-\mathrm{H} 3^{\prime} \mathrm{A}$ & $\mathrm{H3} \cdot \mathrm{A}$ & $\mathrm{HZ} \mathrm{C}^{\prime} \mathrm{A}, \mathrm{HZ} \mathrm{Z}^{\prime} \mathrm{B}$ & 1086 \\
\hline $\mathrm{CEC}$ & $\mathrm{Hz}$ & 6.1161 & $\mathrm{CEC}$ & $H 2^{\prime} A, H Z^{\prime} E-H 3^{\prime} B$ & H3' $\mathrm{B}$ & HZ'A,HZ'B & 5.43 \\
\hline $\operatorname{CEC}$ & $H Z^{\prime} A, H Z^{\prime} B$ & 15557 & $\mathrm{CEC}$ & $\mathrm{HZ}-\mathrm{H} 4{ }^{\prime} \mathrm{A}$ & $\mathrm{Hz}$ & $\mathrm{H} 4{ }^{\prime \mu} \mathrm{A}$ & -001 \\
\hline $\mathrm{CEC}$ & $\mathrm{Hz}$ & 5.4938 & $\mathrm{CEC}$ & $\mathrm{H} 2^{\prime}-\mathrm{H}_{4} \mathrm{~B}$ & $\mathrm{HZ}$ & $\mathrm{H}^{\prime} \mathrm{B}$ & $-0,08$ \\
\hline $\mathrm{CEC}$ & $\mathrm{H} 3^{\prime} \mathrm{A}$ & 13278 & $\mathrm{CEC}$ & H2'-H9'(Me) & $\mathrm{Hz}$ & $\mathrm{HQ}^{\prime}(\mathrm{Me})$ & -0.04 \\
\hline $\operatorname{CEC}$ & H3' $\mathrm{B}$ & 13046 & $\mathrm{CEC}$ & $\mathrm{H} 2-\mathrm{H}^{\prime}$ & $\mathrm{Hz}$ & $H^{\prime}$ & 051 \\
\hline $\operatorname{CEC}$ & $\mathrm{H}_{4}$ & 6.530 & $\mathrm{CEC}$ & $\mathrm{H} 2-\mathrm{HZ}^{\prime}$ & $\mathrm{Hz}$ & $\mathrm{Hz}^{\prime}$ & -001 \\
\hline $\operatorname{CEC}$ & $\mathrm{H} 4^{\prime} \mathrm{A}, \mathrm{H} 4^{\prime} \mathrm{B}$ & 1.2985 & $\operatorname{cEC}$ & $\mathrm{H} 22 \mathrm{~A}-\mathrm{H} 22 \mathrm{~B}$ & $\mathrm{H}^{\prime} \mathrm{A}, \mathrm{H} 4^{\prime} \mathrm{B}$ & $\mathrm{H}_{4}{ }^{\prime} \mathrm{A}, \mathrm{H} 4{ }^{\prime} \mathrm{B}$ & -11.44 \\
\hline $\mathrm{CEC}$ & $\mathrm{H}_{4} \mathrm{AL}_{\mathrm{H}}$ & 1.6462 & $\mathrm{CEC}$ & H2Z4-H5 '(Me) & $\mathrm{H} 4^{\prime} \mathrm{A}, \mathrm{H} 4^{\prime} \mathrm{B}$ & H5 '(Me) & 7.78 \\
\hline $\mathrm{CEC}$ & $\mathrm{H} 4 \mathrm{~B}$ & $1.72 \mathrm{E}$ & $\mathrm{CEC}$ & $H 3{ }^{\prime} A-H 2 Z 4$ & $\mathrm{H} 3^{\prime A \mathrm{~A}}$ & $\mathrm{H} 4^{\prime} \mathrm{A}, \mathrm{H} 4^{\prime} \mathrm{B}$ & 8.49 \\
\hline $\operatorname{CEC}$ & H5 ' (Me) & 08842 & $\mathrm{CEC}$ & $H 3^{\prime} A-H 22 B$ & $\mathrm{H} 3 \mathrm{AH}$ & $\mathrm{H} 4^{\prime} \mathrm{A}, \mathrm{H}_{4} \mathrm{C}^{\prime} \mathrm{E}$ & 6.42 \\
\hline $\operatorname{cex}$ & H5' & 2.1052 & $\mathrm{CEC}$ & $H 3$ 'A-H3'B & $\mathrm{H} 3^{\prime} \mathrm{A}$ & $\mathrm{H} 3^{\prime} \mathrm{B}$ & -1097 \\
\hline $\operatorname{CEC}$ & H5'B & 2.0993 & $\mathrm{CEC}$ & H3 'A-H5 ' (Me) & H3'A & H5 '(Me) & -0.00 \\
\hline $\operatorname{CEC}$ & $\mathrm{H}^{\prime}$ & 5.0056 & $\mathrm{CEC}$ & $\mathrm{H} 3 \mathrm{C}^{\prime} \mathrm{E}-\mathrm{H} 22 \mathrm{~A}$ & H3' $\mathrm{B}$ & $\mathrm{H} 4^{\prime} \mathrm{A}, \mathrm{H} 4^{\prime} \mathrm{B}$ & 6.24 \\
\hline $\mathrm{CEC}$ & $\mathrm{H}^{\prime} \mathrm{O}^{\prime}(\mathrm{Me})$ & 15758 & $\mathrm{CEC}$ & $\mathrm{H} 3$ 'E-H22B & H3' $\mathrm{B}$ & $\mathrm{H} 4^{\prime} \mathrm{A}, \mathrm{H} 4^{\prime} \mathrm{B}$ & 1194 \\
\hline $\operatorname{CEC}$ & H9'(Me) & 13782 & $\operatorname{cEC}$ & H3 'E-H5 '(Me) & H3' $\mathrm{B}$ & H5 '(Me) & 0.04 \\
\hline $\mathrm{H} 2 \mathrm{O}$ & $\mathrm{H} 2 \mathrm{O}$ & 15600 & $\mathrm{CEC}$ & $\mathrm{H}_{4} \mathrm{~A}_{-}-\mathrm{H}_{4} \mathrm{\prime} \mathrm{B}$ & $\mathrm{H} 4 \mathrm{AH}$ & $\mathrm{H}_{4} \mathrm{~B}$ & -14.12 \\
\hline & & & $\operatorname{CEC}$ & H4'A-H5'A & H4'A & H5' & 11.77 \\
\hline & $13 \mathrm{C}$ & & $\mathrm{CEC}$ & H4'A-H5'B & $\mathrm{H} 4{ }^{\prime} \mathrm{AH}$ & H5'B & 5.20 \\
\hline $\operatorname{cEC}$ & 1 & 134.187 & $\mathrm{CEC}$ & $\mathrm{H}_{4} \mathrm{~A}-\mathrm{H} \mathbf{6}^{\prime}$ & $\mathrm{H} 4 \mathrm{AH}$ & $\mathrm{H}^{\prime}$ & -030 \\
\hline $\operatorname{CEC}$ & 5 & 151.1303 & $\mathrm{CEC}$ & H4'E-H5' A & $\mathrm{H}^{\prime} \mathrm{B}$ & H5' & 391 \\
\hline $\operatorname{cEx}$ & 3 & 1448956 & $\mathrm{CEC}$ & H4'E-H5'B & $\mathrm{H}^{\prime} \mathrm{B}$ & H5' $\mathrm{B}$ & 13.21 \\
\hline $\mathrm{CEC}$ & 7 & 131.7643 & $\mathrm{CEC}$ & H4'E- ${ }^{\prime} 6^{\prime}$ & $\mathrm{H} 4 \mathrm{~B}$ & $\mathrm{H} 6^{\prime}$ & -0.21 \\
\hline $\mathrm{CEC}$ & $z^{\prime}$ & 1773652 & $\mathrm{CEC}$ & $\mathrm{H} 4-\mathrm{H}^{\prime}$ & $\mathrm{H}_{4}$ & $H_{1}^{\prime}$ & 0.19 \\
\hline $\mathrm{CEC}$ & $6^{\prime}$ & 1243348 & $\mathrm{CEC}$ & $\mathrm{H} 4-\mathrm{HZ}$ & $\mathrm{H} 4$ & $\mathrm{~Hz}$ & 151 \\
\hline $\mathrm{CEC}$ & $1^{\prime}$ & 1169369 & $\mathrm{CEC}$ & $\mathrm{H} 4-\mathrm{HZ}^{\prime}$ & $\mathrm{H}_{4}$ & $\mathrm{HZ}^{\prime}$ & -002 \\
\hline $\operatorname{cEC}$ & 4 & 109.2606 & $\mathrm{CEC}$ & H5 'A- $\mathrm{HE}$ '(Me) & H5' A & $\mathrm{HS}^{\prime}(\mathrm{Me})$ & 0.68 \\
\hline $\operatorname{CEC}$ & 2 & 1078414 & $\mathrm{CEC}$ & H5 'A-H5'B & H5' A & H5' $\mathrm{B}$ & -14.18 \\
\hline $\mathrm{CEC}$ & 6 & 107.1392 & $\mathrm{CEC}$ & H5 'A- H6' & H5' & $\mathrm{H} 6^{\prime}$ & 691 \\
\hline $\mathrm{CEC}$ & $3^{\prime}$ & 783383 & $\mathrm{CEC}$ & H5 'A- H10'(Me) & H5' 'A & $\mathrm{H}_{10}(\mathrm{Me})$ & 036 \\
\hline $\mathrm{CEC}$ & $4^{\prime}$ & 41.17 & $\mathrm{CEC}$ & H5 'E-HE'(Me) & H5'B & HE '(Me) & 087 \\
\hline $\mathrm{CEC}$ & 1 & 36.0274 & $\mathrm{CEC}$ & H5 'E-H6' & H5' $\mathrm{B}$ & H6' & 738 \\
\hline $\mathrm{CEC}$ & 3 & 31,6007 & $\mathrm{CEC}$ & H5 'E-H10'(Me) & H5'B & $\mathrm{H}^{\prime} 0^{\prime}(\mathrm{Me})$ & 038 \\
\hline $\mathrm{CEC}$ & $z^{\prime}$ & 30.7567 & $\mathrm{CEC}$ & HE '(Me)-H1O'(Me) & $\mathrm{Hs} \mathbf{S}^{\prime}(\mathrm{Me})$ & $\mathrm{H}_{10}(\mathrm{Me})$ & 0,00 \\
\hline $\mathrm{CEC}$ & $g^{\prime}$ & $2636 \mathrm{E}$ & $\mathrm{CEC}$ & $\mathrm{H}^{\prime} \mathrm{O}^{\prime}(\mathrm{Me}) \cdot \mathrm{H} 6^{\prime}$ & $\mathrm{H}_{10}(\mathrm{Me})$ & H6' & -0.77 \\
\hline $\mathrm{CEC}$ & $\mathbf{8}^{\prime}$ & 5.797 & & & & & \\
\hline $\mathrm{CEC}$ & $5^{\prime}$ & 228364 & & & & & \\
\hline $\mathrm{CEC}$ & $4^{\prime}$ & 226615 & & & & & \\
\hline $\mathrm{CEC}$ & $10^{\prime}$ & 17.7348 & & & & & \\
\hline $\mathrm{CEC}$ & $5^{\prime}$ & 14.1383 & & & & & \\
\hline
\end{tabular}


Table S37. The ${ }^{1} \mathrm{H}$ iterative full spin analysis (HiFSA) profiles of CBG (4).

\begin{tabular}{|c|c|c|c|c|c|c|}
\hline Name: & $\mathrm{CBG}$ & & & & & \\
\hline CHEMICA & FTS (ppm) & & COUPLIN & NSTANTS (Hz) & & \\
\hline Spiruystem & Name & $\mathrm{ppm}$ & spirzystem & Shift & Shift & $\mathrm{Hz}$ \\
\hline CBG & $\mathrm{H} 1{ }^{\prime \prime} \mathrm{A}, \mathrm{H} 1$ "B & 2.4536 & CBG & $\mathrm{H} 4, \mathrm{H} 2$ & $\mathrm{H} 4, \mathrm{H} 2$ & 1.80 \\
\hline CBG & H1'A, H1'B & 3.3903 & CBG & $\mathrm{H} 1$ "A, $\mathrm{H} 1$ "B & $\mathrm{H} 1{ }^{\prime \prime A, H 1 " B}$ & -13.28 \\
\hline CBG & $\mathrm{H} 1, \mathrm{H} 5$ & 4.9645 & CBG & $\mathrm{H} 1$ "A, $\mathrm{H} 1$ "B & $\mathrm{H} 2^{\prime \prime} \mathrm{A}, \mathrm{H} 2^{\prime \prime} \mathrm{B}$ & 9.36 \\
\hline CBG & H10'(Me) & 1.5897 & CBG & $\mathrm{H} 1$ "A, H1"B & $\mathrm{H} 2^{\prime \prime} \mathrm{A}, \mathrm{H} 2^{\prime \prime} \mathrm{B}$ & 6.17 \\
\hline CBG & $\mathrm{H} 2{ }^{\prime \prime} \mathrm{A}, \mathrm{H} 2^{\prime \prime} \mathrm{B}$ & 1.5837 & CBG & $\mathrm{H} 2^{\prime}$ & $\mathrm{H} 4{ }^{\prime} \mathrm{A}, \mathrm{H} 4{ }^{\prime} \mathrm{B}$ & -1.44 \\
\hline CBG & $\mathrm{H} 2^{\prime}$ & 5.2701 & CBG & $\mathrm{H} 2^{\prime}$ & $\mathrm{H} 1^{\prime} \mathrm{A}, \mathrm{H} 1^{\prime} \mathrm{B}$ & 7.10 \\
\hline CBG & H3"A,H3"B & 1.3210 & CBG & $\mathrm{H} 2^{\prime}$ & H9'(Me) & -1.10 \\
\hline CBG & $\mathrm{H} 4{ }^{\prime \prime} \mathrm{A}, \mathrm{H} 4{ }^{\prime \prime B}$ & 1.2974 & CBG & $\mathrm{H} 2^{\prime \prime} \mathrm{A}, \mathrm{H} 2^{\prime \prime} \mathrm{B}$ & $\mathrm{H} 2^{\prime \prime} \mathrm{A}, \mathrm{H} 2{ }^{\prime \prime} \mathrm{B}$ & -12.28 \\
\hline CBG & H4'A, H4'B & 2.0602 & CBG & $\mathrm{H} 2{ }^{\prime \prime} \mathrm{A}, \mathrm{H} 2^{\prime \prime} \mathrm{B}$ & H3"A,H3"B & 7.93 \\
\hline CBG & $\mathrm{H}_{4}, \mathrm{H} 2$ & 6.2478 & CBG & $\mathrm{H} 2^{\prime \prime} \mathrm{A}, \mathrm{H} 2{ }^{\prime \prime} \mathrm{B}$ & H3"A,H3"B & 7.51 \\
\hline CBG & H5"'(Me) & 0.8853 & CBG & H3"A,H3"B & $\mathrm{H} 3{ }^{\prime \prime A, H}, \mathrm{H}$ "B & -13.03 \\
\hline CBG & $\mathrm{H} 5^{\prime} \mathrm{A}, \mathrm{H} 5^{\prime} \mathrm{B}$ & 2.1035 & CBG & H3"A,H3"B & $\mathrm{H} 4$ "A, $\mathrm{H} 4$ "B & 9.64 \\
\hline $\mathrm{CBG}$ & $\mathrm{H} 6^{\prime}$ & 5.0528 & $\mathrm{CBG}$ & H3"A,H3"B & $\mathrm{H} 4 " \mathrm{~A}, \mathrm{H} 4{ }^{\prime \prime} \mathrm{B}$ & 4.78 \\
\hline CBG & $\mathrm{H} 8$ '(Me) & 1.6760 & CBG & $\mathrm{H} 4 " \mathrm{~A}, \mathrm{H} 4$ "B & $\mathrm{H} 4{ }^{\prime \prime} \mathrm{A}, \mathrm{H} 4$ "B & -12.38 \\
\hline CBG & H9'(Me) & 1.8100 & CBG & $\mathrm{H} 4{ }^{\prime \prime} \mathrm{A}, \mathrm{H} 4{ }^{\prime \prime} \mathrm{B}$ & H5'(Me) & 7.14 \\
\hline $\mathrm{H} 2 \mathrm{O}$ & $\mathrm{H} 2 \mathrm{O}$ & 1.5579 & CBG & H5'A, H5'B & H10'(Me) & 0.49 \\
\hline & & & CBG & $\mathrm{H} 5^{\prime} \mathrm{A}, \mathrm{H} 5^{\prime} \mathrm{B}$ & $\mathrm{H} 5^{\prime} \mathrm{A}, \mathrm{H} 5^{\prime} \mathrm{B}$ & -13.44 \\
\hline & $13 \mathrm{C}$ & $\mathrm{ppm}$ & $\mathrm{CBG}$ & $\mathrm{H} 5^{\prime} \mathrm{A}, \mathrm{H} 5^{\prime} \mathrm{B}$ & $\mathrm{H} 6^{\prime}$ & 6.53 \\
\hline & 1 & 1549569 & CBG & $\mathrm{H} 5^{\prime} \mathrm{A}, \mathrm{H} 5^{\prime} \mathrm{B}$ & $\mathrm{H}^{\prime}(\mathrm{Me})$ & 1.00 \\
\hline & 5 & 1549569 & $\mathrm{CBG}$ & $\mathrm{H} 4 \mathrm{~A}, \mathrm{H} 4$ 'B & H5'A, H5'B & 5.63 \\
\hline & 3 & 142.9181 & CBG & H4'A, H4'B & H5'A, H5'B & 8.93 \\
\hline & $3^{\prime}$ & 139.1569 & CBG & $\mathrm{H} 4{ }^{\prime} \mathrm{A}, \mathrm{H} 4{ }^{\prime} \mathrm{B}$ & $\mathrm{H} 4 \mathrm{~A}^{\prime}, \mathrm{H} 4{ }^{\prime} \mathrm{B}$ & -13.16 \\
\hline & $7^{\prime}$ & 132.2154 & CBG & $\mathrm{H}^{\prime} \mathrm{A}, \mathrm{H} 4^{\prime} \mathrm{B}$ & H9'(Me) & -0.17 \\
\hline & $6^{\prime}$ & 123.8989 & CBG & $\mathrm{H} 4{ }^{\prime} \mathrm{A}, \mathrm{H} 4{ }^{\prime} \mathrm{B}$ & $\mathrm{H} 6^{\prime}$ & 0.00 \\
\hline & $2^{\prime}$ & 121.8321 & $\mathrm{CBG}$ & $\mathrm{H} 6^{\prime}$ & H10'(Me) & -1.39 \\
\hline & 6 & 110.6944 & CBG & $\mathrm{H} 6^{\prime}$ & H8'(Me) & -1.44 \\
\hline & 2 & 108.5095 & $\mathrm{CBG}$ & H8'(Me) & H10'(Me) & -0.01 \\
\hline & 4 & 108.4281 & CBG & H1'A, H1'B & H1'A, H1'B & -18.32 \\
\hline & $4^{\prime}$ & 39.8402 & CBG & H9'(Me) & $\mathrm{H} 1{ }^{\prime} \mathrm{A}, \mathrm{H} 1^{\prime} \mathrm{B}$ & 1.02 \\
\hline & $1^{\prime \prime}$ & 35.6523 & & & & \\
\hline & $3 "$ & 31.632 & & & & \\
\hline & $2^{\prime \prime}$ & 30.938 & & & & \\
\hline & $5^{\prime}$ & 26.7293 & & & & \\
\hline & $8^{\prime}$ & 25.8206 & & & & \\
\hline & $1^{\prime}$ & 22.6897 & & & & \\
\hline & $4 "$ & 22.3993 & & & & \\
\hline & $10^{\prime}$ & 17.8447 & & & & \\
\hline & $9^{\prime}$ & 16.3366 & & & & \\
\hline & $5^{\prime \prime}$ & 14.1678 & & & & \\
\hline
\end{tabular}


Table S38. The ${ }^{1} \mathrm{H}$ iterative full spin analysis (HiFSA) profiles of CBN (5).

\begin{tabular}{|c|c|c|c|c|c|c|c|}
\hline Name: & $\mathrm{CBN}$ & & & & & & \\
\hline CHEMICA & HIFTS (ppm) & & COUPLIN & ONSTANTS (Hz) & & & \\
\hline Spireystrem & Name & $\mathrm{ppm}$ & spirrystem m & Name & Shift & Shift & $\mathrm{Hz}$ \\
\hline $\mathrm{CBN}$ & $1(\mathrm{OH})$ & 5.3012 & $\mathrm{CBN}$ & $\mathrm{H} 1^{\prime} \mathrm{A}-\mathrm{H} 1^{\prime} \mathrm{B}$ & H1'A, H1'B & $\mathrm{H}^{\prime} \mathrm{A}, \mathrm{H} 1^{\prime} \mathrm{B}$ & -13.12 \\
\hline $\mathrm{CBN}$ & H1'A,H1'B & 2.4986 & $\mathrm{CBN}$ & $\mathrm{H} 1^{\prime} \mathrm{A}-\mathrm{H}_{2} \mathrm{~A}$ & H1'A,H1'B & $\mathrm{H} 2^{\prime} \mathrm{A}, \mathrm{H}_{2}^{\prime} \mathrm{B}$ & 9.27 \\
\hline $\mathrm{CBN}$ & $\mathrm{H} 10$ & 8.1857 & CBN & $\mathrm{H}^{\prime} \mathrm{A}_{-} \mathrm{H}_{2}{ }^{\prime} \mathrm{B}$ & H1'A,H1'B & $\mathrm{H} 2^{\prime} \mathrm{A}, \mathrm{H}_{2}{ }^{\prime} \mathrm{B}$ & 6.23 \\
\hline $\mathrm{CBN}$ & H11(Me) & 2.3968 & CBN & $\mathrm{H} 10-\mathrm{H} 8$ & $\mathrm{H} 10$ & $\mathrm{H} 8$ & 1.81 \\
\hline $\mathrm{CBN}$ & H12(Me),H13(Me) & 1.6151 & $\mathrm{CBN}$ & H11(Me)-H10 & H11(Me) & $\mathrm{H} 10$ & -0.65 \\
\hline $\mathrm{CBN}$ & $\mathrm{H} 2$ & 6.2831 & CBN & H11(Me)-H7 & H11(Me) & $\mathrm{H7}$ & 0.03 \\
\hline $\mathrm{CBN}$ & $\mathrm{H} 2^{\prime} \mathrm{A}, \mathrm{H} 2^{\prime} \mathrm{B}$ & 1.5998 & $\mathrm{CBN}$ & $\mathrm{H} 11$ (Me)-H8 & H11(Me) & $\mathrm{H} 8$ & -0.69 \\
\hline $\mathrm{CBN}$ & H3'A, H3'B & 1.3221 & $\mathrm{CBN}$ & $\mathrm{H} 2^{\prime} \mathrm{A}_{-} \mathrm{H}_{2}{ }^{\prime} \mathrm{B}$ & $\mathrm{H}^{\prime}{ }^{\prime} \mathrm{A}, \mathrm{H} 2^{\prime} \mathrm{B}$ & $\mathrm{H}^{\prime}{ }^{\prime} \mathrm{A}, \mathrm{H} 2^{\prime} \mathrm{B}$ & -13.27 \\
\hline $\mathrm{CBN}$ & $\mathrm{H} 4$ & 6.4552 & CBN & H2'A-H3'A & $\mathrm{H}^{\prime}{ }^{\prime} \mathrm{A}, \mathrm{H} 2^{\prime} \mathrm{B}$ & H3'A, H3'B & 7.40 \\
\hline $\mathrm{CBN}$ & $\mathrm{H} 4^{\prime} \mathrm{A}, \mathrm{H} 4^{\prime} \mathrm{B}$ & 1.3400 & $\mathrm{CBN}$ & $\mathrm{H} 2^{\prime} \mathrm{A}_{-} \mathrm{H} 3 \mathrm{~B}^{\prime} \mathrm{B}$ & $\mathrm{H}^{\prime}{ }^{\prime} \mathrm{A}, \mathrm{H} 2^{\prime} \mathrm{B}$ & H3'A,H3'B & 0.00 \\
\hline $\mathrm{CBN}$ & H5'(Me) & 0.9036 & $\mathrm{CBN}$ & H3'A-H3'B & H3'A, H3'B & H3'A, H3'B & -14.60 \\
\hline $\mathrm{CBN}$ & $\mathrm{H} 7$ & 7.1563 & $\mathrm{CBN}$ & $\mathrm{H} 3{ }^{\prime} \mathrm{A}-\mathrm{H}^{\prime} \mathrm{A}$ & H3'A, H3'B & $\mathrm{H}^{\prime} \mathrm{A}, \mathrm{H} 4{ }^{\prime} \mathrm{B}$ & 4.93 \\
\hline $\mathrm{CBN}$ & $\mathrm{H} 8$ & 7.0813 & $\mathrm{CBN}$ & $\mathrm{H} 3{ }^{\prime} \mathrm{A}-\mathrm{H} 4^{\prime} \mathrm{B}$ & H3'A,H3'B & $\mathrm{H}_{4} \mathrm{~A}^{\prime}, \mathrm{H} 4{ }^{\prime} \mathrm{B}$ & 5.44 \\
\hline $\mathrm{H} 2 \mathrm{O}$ & $\mathrm{H} 2 \mathrm{O}$ & 1.7282 & $\mathrm{CBN}$ & H3'A-H5'(Me) & H3'A, H3'B & H5'(Me) & 0.00 \\
\hline & & & CBN & $\mathrm{H}^{\prime}{ }^{\prime} \mathrm{A}-\mathrm{H} 4^{\prime} \mathrm{B}$ & $\mathrm{H}_{4} \mathrm{~A}^{\mathrm{A}, \mathrm{H}}$ ''B $^{\prime}$ & $\mathrm{H}_{4}^{\prime} \mathrm{A}, \mathrm{H} 4{ }^{\prime} \mathrm{B}$ & -11.78 \\
\hline & $13 \mathrm{C}$ & $\mathrm{ppm}$ & $\mathrm{CBN}$ & H4'A-H5'(Me) & $\mathrm{H}^{\prime} \mathrm{A}^{\prime}, \mathrm{H} 4{ }^{\prime} \mathrm{B}$ & H5'(Me) & 7.14 \\
\hline $\mathrm{CBN}$ & 1 & 154.7159 & $\mathrm{CBN}$ & $\mathrm{H} 4 \mathrm{H} 2$ & $\mathrm{H} 4$ & $\mathrm{H} 2$ & 1.63 \\
\hline $\mathrm{CBN}$ & 5 & 153.1846 & $\mathrm{CBN}$ & $\mathrm{H} 7-\mathrm{H} 10$ & $\mathrm{H}_{7}$ & $\mathrm{H} 10$ & 0.22 \\
\hline $\mathrm{CBN}$ & 3 & 144.6768 & $\mathrm{CBN}$ & $\mathrm{H} 7-\mathrm{H} 8$ & $\mathrm{H}_{7}$ & $\mathrm{H} 8$ & 7.84 \\
\hline $\mathrm{CBN}$ & $6 a$ & 137.0308 & & & & & \\
\hline $\mathrm{CBN}$ & 9 & 136.9873 & & & & & \\
\hline $\mathrm{CBN}$ & 8 & 127.6644 & & & & & \\
\hline $\mathrm{CBN}$ & 10 & 126.5514 & & & & & \\
\hline $\mathrm{CBN}$ & 7 & 122.7475 & & & & & \\
\hline $\mathrm{CBN}$ & $10 \mathrm{~b}, 4$ & 110.8925 & & & & & \\
\hline $\mathrm{CBN}$ & $10 b, 4$ & 110.8925 & & & & & \\
\hline $\mathrm{CBN}$ & 2 & 110.0289 & & & & & \\
\hline $\mathrm{CBN}$ & $10 a$ & 108.8335 & & & & & \\
\hline $\mathrm{CBN}$ & 6 & 77.4942 & & & & & \\
\hline $\mathrm{CBN}$ & $1^{\prime \prime}$ & 35.7244 & & & & & \\
\hline $\mathrm{CBN}$ & $3^{\prime \prime}$ & 31.5991 & & & & & \\
\hline $\mathrm{CBN}$ & $2^{\prime \prime}$ & 30.5759 & & & & & \\
\hline $\mathrm{CBN}$ & 12,13 & 27.2232 & & & & & \\
\hline $\mathrm{CBN}$ & 12,13 & 27.2232 & & & & & \\
\hline $\mathrm{CBN}$ & $4^{\prime \prime}$ & 22.6611 & & & & & \\
\hline $\mathrm{CBN}$ & 11 & 21.6534 & & & & & \\
\hline $\mathrm{CBN}$ & $5^{\prime \prime}$ & 14.1522 & & & & & \\
\hline
\end{tabular}


Table S39. The ${ }^{1} \mathrm{H}$ iterative full spin analysis (HiFSA) profiles of CBDV (6).

\begin{tabular}{|c|c|c|c|c|c|c|c|}
\hline \multicolumn{8}{|c|}{ Name: CBDy } \\
\hline \multicolumn{3}{|c|}{ CHEMICALSHIFTS (ppm) } & \multicolumn{5}{|c|}{ COUPLING CONSTANTS (Hz) } \\
\hline Spirsystem & Name & $\mathrm{ppm}$ & Spireystem m & Name & Shift & Shift & $\mathrm{Hz}$ \\
\hline CBDV & $\mathrm{H} 1$ & 3.8420 & CBDV & $\mathrm{H} 1{ }^{\prime \prime} \mathrm{A}, \mathrm{H} 1$ "B-H2"A,H2"B & $\mathrm{H} 1$ "A, H1"B & $\mathrm{H} 2^{\prime \prime} \mathrm{A}, \mathrm{H} 2^{\prime \prime} \mathrm{B}$ & 7.60 \\
\hline CBDV & $\mathrm{H} 1{ }^{\prime \prime}, \mathrm{H} 1$ "B & 2.4265 & CBDV & H1"A,H1"B-H3"(Me) & H3"(Me) & $\mathrm{H} 1{ }^{\prime \prime} \mathrm{A}, \mathrm{H} 1$ "B & 0.01 \\
\hline CBDV & H1'(OH) & 5.9652 & CBDV & $\mathrm{H} 1-\mathrm{H} 2^{\prime}$ & $\mathrm{H} 1$ & $\mathrm{H} 2^{\prime}$ & 0.43 \\
\hline CBDV & H10(Me) & 1.6534 & CBDV & $\mathrm{H} 1-\mathrm{H} 4^{\prime}$ & $\mathrm{H} 1$ & $\mathrm{H}^{\prime}$ & 0.43 \\
\hline CBDV & $\mathrm{H} 2$ & 5.5702 & CBDV & H10(Me)-H9A & H10(Me) & $\mathrm{H} 9 \mathrm{~A}$ & -1.42 \\
\hline CBDV & $\mathrm{H} 2^{\prime \prime} \mathrm{A}, \mathrm{H} 2^{\prime \prime} \mathrm{B}$ & 1.5856 & CBDV & $\mathrm{H} 10$ (Me) $\mathrm{HgB}$ & H10(Me) & $\mathrm{H} 9 \mathrm{~B}$ & -0.40 \\
\hline CBDV & $\mathrm{H} 2^{\prime}$ & 6.2683 & CBDV & $\mathrm{H} 2 " \mathrm{~A}, \mathrm{H} 2$ "B-H3"(Me) & H3'(Me) & $\mathrm{H} 2^{\prime \prime} \mathrm{A}, \mathrm{H} 2^{\prime \prime} \mathrm{B}$ & 7.35 \\
\hline CBDV & H3"(Me) & 0.9032 & CBDV & $\mathrm{H}^{\prime}-\mathrm{H} 6$ & $\mathrm{H} 6$ & $\mathrm{H} 2^{\prime}$ & -0.88 \\
\hline CBDV & $\mathrm{H}^{\prime}$ & 6.1786 & CBDV & $\mathrm{H} 2-\mathrm{H} 1$ & $\mathrm{H} 2$ & $\mathrm{H} 1$ & 1.55 \\
\hline CBDV & H4A. & 2.2329 & CBDV & $\mathrm{H} 2-\mathrm{H} 4 \mathrm{~A}$ & $\mathrm{H} 2$ & H4A. & -0.38 \\
\hline CBDV & $\mathrm{H} 4 \mathrm{~B}$ & 2.0974 & CBDV & $\mathrm{H} 2-\mathrm{H} 4 \mathrm{~B}$ & $\mathrm{H} 2$ & $\mathrm{H} 4 \mathrm{~B}$ & 0.39 \\
\hline CBDV & H5'(OH) & 4.5878 & CBDV & $\mathrm{H} 2-\mathrm{H} 7(\mathrm{Me})$ & $\mathrm{H} 2$ & H7(Me) & -0.56 \\
\hline CBDV & H5A. & 1.7810 & CBDV & $\mathrm{H} 4^{\prime}-\mathrm{H} 6$ & $\mathrm{H} 6$ & $\mathrm{H} 4^{\prime}$ & -0.37 \\
\hline CBDV & $\mathrm{H} 5 \mathrm{~B}$ & 1.8367 & CBDV & $\mathrm{H} 4 \mathrm{~A}_{-} \mathrm{H} 1$ & $\mathrm{H} 4 \mathrm{~A}$ & $\mathrm{H} 1$ & 3.38 \\
\hline CBDV & $\mathrm{H} 6$ & 2.3938 & CBDV & H4A-H5A. & H4A & $\mathrm{H} 5 \mathrm{~A}$ & 11.71 \\
\hline CBDV & H7(Me) & 1.7933 & CBDV & $\mathrm{H} 4 \mathrm{~A}-\mathrm{H} 5 \mathrm{~B}$ & $\mathrm{H} 4 \mathrm{~A}$ & $\mathrm{H} 5 \mathrm{~B}$ & 5.85 \\
\hline CBDV & $\mathrm{H} 9 \mathrm{~A}$ & 4.6650 & CBDV & $\mathrm{H} 4 \mathrm{~A}-\mathrm{H} 7(\mathrm{Me})$ & H4A & H7(Me) & -0.61 \\
\hline CBDV & $\mathrm{H} 9 \mathrm{~B}$ & 4.5610 & CBDV & $\mathrm{H} 4 \mathrm{~B}-\mathrm{H} 1$ & $\mathrm{H} 4 \mathrm{~B}$ & $\mathrm{H} 1$ & 2.67 \\
\hline \multirow[t]{3}{*}{$\mathrm{H} 2 \mathrm{O}$} & $\mathrm{H} 2 \mathrm{O}$ & 1.6857 & CBDY & $\mathrm{H} 4 \mathrm{~B}-\mathrm{H} 4 \mathrm{~A}$ & $\mathrm{H} 4 \mathrm{~B}$ & H4A. & -17.95 \\
\hline & & & CBDV & H4B-H5A & $\mathrm{H} 4 \mathrm{~B}$ & H5A & 5.18 \\
\hline & $13 \mathrm{C}$ & $\mathrm{ppm}$ & CBDV & $\mathrm{H} 4 \mathrm{~B}-\mathrm{H} 5 \mathrm{~B}$ & $\mathrm{H} 4 \mathrm{~B}$ & $\mathrm{H} 5 \mathrm{~B}$ & 2.16 \\
\hline CBDV & $1^{\prime}$ & 156.0116 & CBDV & H4B-H7(Me) & $\mathrm{H} 4 \mathrm{~B}$ & H7(Me) & -0.30 \\
\hline CBDV & $5^{\prime}$ & 153.7236 & CBDV & $\mathrm{H} 5 \mathrm{~A}-\mathrm{H} 5 \mathrm{~B}$ & H5A & $\mathrm{H} 5 \mathrm{~B}$ & -14.31 \\
\hline CBDV & 8 & 149.6005 & CBDV & $\mathrm{H} 6-\mathrm{H} 1$ & $\mathrm{H} 6$ & $\mathrm{H} 1$ & 10.47 \\
\hline CBDV & $3^{\prime}$ & 142.9353 & CBDV & $\mathrm{H} 6-\mathrm{H} 10(\mathrm{Me})$ & $\mathrm{H} 6$ & H10(Me) & -0.07 \\
\hline CBDV & 3 & 140.2473 & CBDV & $\mathrm{H} 6-\mathrm{H} 2$ & $\mathrm{H} 6$ & $\mathrm{H} 2$ & 0.24 \\
\hline CBDV & 2 & 124.2339 & CBDV & $\mathrm{H} 6-\mathrm{H} 5 \mathrm{~A}$ & $\mathrm{H} 6$ & H5A. & 12.34 \\
\hline CBDV & $6^{\prime}$ & 113.921 & CBDV & H6-H5B & $\mathrm{H} 6$ & $\mathrm{H} 5 \mathrm{~B}$ & 2.98 \\
\hline CBDV & 9 & 110.9781 & CBDV & $\mathrm{H} 6-\mathrm{H} 9 \mathrm{~A}$ & H6 & $\mathrm{H} 9 \mathrm{~A}$ & -0.46 \\
\hline CBDV & $2^{\prime}$ & 110.2509 & CBDV & H6-H9B & $\mathrm{H} 6$ & $\mathrm{H} 9 \mathrm{~B}$ & -0.11 \\
\hline CBDV & $4^{\prime}$ & 108.1496 & CBDV & $\mathrm{H} 7(\mathrm{Me}) \mathrm{H} 1$ & $\mathrm{H} 7(\mathrm{Me})$ & $\mathrm{H} 1$ & 1.32 \\
\hline CBDV & 6 & 46.2713 & CBDV & H9A-H9B & H9A & H9B & 0.95 \\
\hline CBDV & 1 & 37.7068 & & & & & \\
\hline CBDV & $1 "$ & 37.4399 & & & & & \\
\hline CBDV & 4 & 30.4463 & & & & & \\
\hline CBDV & 5 & 28.5451 & & & & & \\
\hline CBDV & 7 & 24.1685 & & & & & \\
\hline CBDV & $2^{\prime \prime}$ & 23.5891 & & & & & \\
\hline CBDV & 10 & 20.7092 & & & & & \\
\hline CBDV & $3^{\prime \prime}$ & 13.873 & & & & & \\
\hline
\end{tabular}

